ESAIM: COCV 21 (2015) 835-856

DOI: $10.1051 / \mathrm{cocv} / 2014052$
ESAIM: Control, Optimisation and Calculus of Variations

www.esaim-cocv.org

\title{
STACKELBERG-NASH EXACT CONTROLLABILITY FOR LINEAR AND SEMILINEAR PARABOLIC EQUATIONS ${ }^{* * * *}$
}

\author{
F.D. Araruna ${ }^{1}$, E. Fernández-Cara ${ }^{2}$ and M.C. Santos ${ }^{1,3}$
}

\begin{abstract}
This paper deals with the application of Stackelberg-Nash strategies to the control of parabolic equations. We assume that we can act on the system through a hierarchy of controls. A first control (the leader) is assumed to choose the policy. Then, a Nash equilibrium pair (corresponding to a noncooperative multiple-objective optimization strategy) is found; this governs the action of the other controls (the followers). The main novelty in this paper is that, this way, we can obtain the exact controllability to a prescribed (but arbitrary) trajectory. We study linear and semilinear problems and, also, problems with pointwise constraints on the followers.
\end{abstract}

Mathematics Subject Classification. 34K35, 49J20, 35K10.

Received June 27, 2014. Revised September 27, 2014

Published online May 20, 2015.

\section{INTRODUCTION}

In classical control theory, we usually find a state equation or system and one control with the mission of achieving a predetermined goal. Frequently (but not always), the goal is to minimize a cost functional in a prescribed family of admissible controls.

A more interesting situation arises when several (in general, conflictive or contradictory) objectives are considered. This may happen, for example, if the cost function is the sum of several terms and it is not clear how to average. It can also be expectable to have more than one control acting on the equation. In these cases, we are led to consider multi-objective control problems.

In contrast with the mono-objective case, various strategies for the choice of good controls can appear, depending of the characteristics of the problem. Moreover, these strategies can be cooperative (when the controls mutually cooperate in order to achieve some goals) or noncooperative.

\footnotetext{
Keywords and phrases. Controllability, Stackelberg-Nash strategies, Carleman inequalities.

* Partially supported by INCTMat, CAPES, CNPq (Brasil) and MathAmSud COSIP.

** Partially supported by grant MTM2010-15592 (DGI-MICINN, Spain) and CAPES (Brasil).

1 Dpto. de Matemática, Universidade Federal da Paraíba, 58051-900 João Pessoa - PB, Brasil. fagner@mat.ufpb.br

2 Dpto. EDAN and IMUS, University of Sevilla, Aptdo. 1160, 41080 Sevilla, Spain. cara@us.es

3 Dpto. de Matemática, Universidade Federal de Pernambuco, 50740-540 Recife-PE, Brasil. mauricio@dmat.ufpe.br
} 
There exist several equilibrium concepts for multi-objective problems, with origin in game theory, mainly motivated by economics. Each of them determines a strategy. Thus, let us mention the noncooperative optimization strategy proposed by Nash [16], the Pareto cooperative strategy [17] and the Stackelberg hierarchical-cooperative strategy [21].

In the context of the control of PDEs, a relevant question is whether one is able to steer the system to a desired state (exactly or approximately) by applying controls that correspond to one of these strategies. Up to date, there has been some work on the subject:

- The papers by Lions $[14,15]$, where the author gives some results concerning Pareto and Stackelberg strategies, respectively.

- The paper by Díaz and Lions [4], where the approximate controllability of a system is established following a Stackelberg-Nash strategy and the extension in Díaz [3], that provides a characterization of the solution by means of Fenchel-Rockafellar duality theory.

- The papers $[18,19]$, where Ramos et al. study Nash equilibria from the theoretical and numerical viewpoints for linear parabolic PDEs and for the Burgers equation.

- Finally, let us mention that the Stackelberg-Nash strategy for the Stokes systems has been studied by Guillén-González et al. in [11].

The controllability issues considered in these works only provide answers at the approximate level. This means that the main results assert that one can lead the system to a state that is arbitrarily close (but not identical) to a desired target.

The main novelty of the present paper is to extend the analysis and the results to an exact controllability framework.

\subsection{The problems and their motivations}

Let $\Omega \subset \mathbb{R}^{N}$ be a bounded domain whose boundary $\Gamma$ is regular enough. Let $T>0$ be given and let us consider the cylinder $Q=\Omega \times(0, T)$, with lateral boundary $\Sigma=\Gamma \times(0, T)$. In the sequel, we will denote by $C$ a generic positive constant. Sometimes, we will indicate the data on which $C$ depends by writing $C(\Omega), C(\Omega, T)$, etc. The usual norm and scalar product in $L^{2}(\Omega)$ will be respectively denoted by $\|\cdot\|$ and $(\cdot, \cdot)$.

We are interested in the proof of the exact controllability to the trajectories of a multi-objective parabolic PDE problem in $Q$, where we apply a Stackelberg-Nash strategy. For simplicity, we will assume that only three controls are applied (one leader and two followers), but very similar considerations hold for systems with a higher number of controls.

We will consider systems of the form

$$
\left\{\begin{array}{lr}
y_{t}-\Delta y+a(x, t) y=F(y)+f 1_{\mathcal{O}}+v^{1} 1_{\mathcal{O}_{1}}+v^{2} 1_{\mathcal{O}_{2}} & \text { in } Q, \\
y=0 & \text { on } \Sigma, \\
y(\cdot, 0)=y^{0} & \text { in } \Omega,
\end{array}\right.
$$

where $y=y(x, t)$ is the state, $a \in L^{\infty}(Q), F$ is a locally Lipschitz-continuous function and $y^{0}$ is prescribed. In (1.1), the set $\mathcal{O} \subset \Omega$ is the main control domain and $\mathcal{O}_{1}, \mathcal{O}_{2} \subset \Omega$ are the secondary control domains (all them are supposed to be small); $1_{\mathcal{O}}, 1_{\mathcal{O}_{1}}$ and $1_{\mathcal{O}_{2}}$ are the characteristic functions of $\mathcal{O}, \mathcal{O}_{1}$ and $\mathcal{O}_{2}$, respectively; the controls are $f, v^{1}$ and $v^{2}$, where $f$ is the leader and $v^{1}$ and $v^{2}$ are the followers.

Let $\mathcal{O}_{1, d}, \mathcal{O}_{2, d} \subset \Omega$ be open sets, representing observation domains for the followers. We will consider the (secondary) functionals

$$
J_{i}\left(f ; v^{1}, v^{2}\right):=\frac{\alpha_{i}}{2} \iint_{\mathcal{O}_{i, d} \times(0, T)}\left|y-y_{i, d}\right|^{2} \mathrm{~d} x \mathrm{~d} t+\frac{\mu_{i}}{2} \iint_{\mathcal{O}_{i} \times(0, T)}\left|v^{i}\right|^{2} \mathrm{~d} x \mathrm{~d} t, \quad i=1,2
$$

and the main functional

$$
J(f):=\frac{1}{2} \iint_{\mathcal{O} \times(0, T)}|f|^{2} \mathrm{~d} x \mathrm{~d} t,
$$

where the $\alpha_{i}>0, \mu_{i}>0$ are constants and the $y_{i, d}=y_{i, d}(x, t)$ are given functions. 
The control process can be described as follows:

1. The followers $v^{1}$ and $v^{2}$ assume that the leader $f$ has made a choice and intend to be a Nash equilibrium for the costs $J_{i}(i=1,2)$.

Thus, once $f$ has been fixed, we look for controls $v^{i} \in L^{2}\left(\mathcal{O}_{i} \times(0, T)\right)$ that satisfy

$$
J_{1}\left(f ; v^{1}, v^{2}\right)=\min _{\hat{v}^{1}} J_{1}\left(f ; \hat{v}^{1}, v^{2}\right), \quad J_{2}\left(f ; v^{1}, v^{2}\right)=\min _{\hat{v}^{2}} J_{2}\left(f ; v^{1}, \hat{v}^{2}\right) .
$$

Any pair $\left(v^{1}, v^{2}\right)$ satisfying (1.4) is called a Nash equilibrium for $J_{1}$ and $J_{2}$.

Note that, if the functionals $J_{i}(i=1,2)$ are convex, then $\left(v^{1}, v^{2}\right)$ is a Nash equilibrium if and only if

$$
J_{1}^{\prime}\left(f ; v^{1}, v^{2}\right)\left(\hat{v}^{1}, 0\right)=0, \quad \forall \hat{v}^{1} \in L^{2}\left(\mathcal{O}_{1} \times(0, T)\right), \quad v^{1} \in L^{2}\left(\mathcal{O}_{1} \times(0, T)\right)
$$

and

$$
J_{2}^{\prime}\left(f ; v^{1}, v^{2}\right)\left(0, \hat{v}^{2}\right)=0, \quad \forall \hat{v}^{2} \in L^{2}\left(\mathcal{O}_{2} \times(0, T)\right), \quad v^{2} \in L^{2}\left(\mathcal{O}_{2} \times(0, T)\right) .
$$

2. Let us fix an uncontrolled trajectory of (1.1), that is, a sufficiently regular solution to the system

$$
\begin{cases}\bar{y}_{t}-\Delta \bar{y}+a(x, t) \bar{y}=F(\bar{y}) & \text { in } Q, \\ \bar{y}=0 & \text { on } \Sigma, \\ \bar{y}(\cdot, 0)=\bar{y}^{0} & \text { in } \Omega .\end{cases}
$$

Once the Nash equilibrium has been identified and fixed for each $f$, we look for a control $\hat{f} \in L^{2}(\mathcal{O} \times(0, T))$ such that

$$
J(\hat{f})=\min _{f} J(f)
$$

subject to the restriction of exact controllability

$$
y(\cdot, T)=\bar{y}(\cdot, T) \quad \text { in } \quad \Omega .
$$

Several motivations can be found for control problems of this kind:

- If $y=y(x, t)$ is viewed as a temperature distribution in a body, we interpret that our intention is to drive $y$ to a desired $\bar{y}$ at time $T$ by heating and cooling (acting only on the small subdomains $\mathcal{O}, \mathcal{O}_{1}$ and $\mathcal{O}_{2}$ ), trying at the same time to keep reasonable temperatures in $\mathcal{O}_{1, d}$ and $\mathcal{O}_{2, d}$ during the whole time interval $(0, T)$.

- The same control strategy makes sense in the context of fluid mechanics. Thus, we can replace (1.1) and (1.7) by similar Stokes and/or Navier-Stokes systems and we can look for controls $\bar{f}$ and associated Nash equilibria $\left(v_{1}, v_{2}\right)$ satisfying (1.8)-(1.9). In this case, it is assumed that we act on the system through mechanical forces applied on $\mathcal{O}, \mathcal{O}_{1}$ and $\mathcal{O}_{2}$ and the goal is to reach $\bar{y}$ at time $T$ keeping the velocity field $y$ not too far from $y_{i, d}$ in $\mathcal{O}_{i, d} \times(0, T)(i=1,2)$.

- In the framework of mathematical finance, this can also be an interesting question. For instance, it is well known that the price of an European call option is governed by a backward PDE similar to (1.1). Now, the independent variable $x$ must be interpreted as the stock price and $t$ is in fact the reverse of time (we fix a situation at $t=T$ and we want to know what to do in order to arrive at this situation from a well chosen state). In this regard, it can be interesting to control the solution of the system with the composed action of several agents, each of them corresponding to a different range of values of $x$. For further information on the modeling and control of phenomena of this kind, see for instance [2,20,22]. 


\subsection{The main results}

We will have to impose the following assumption:

$$
\mathcal{O}_{1, d}=\mathcal{O}_{2, d}
$$

Accordingly, we will denote these sets by $\mathcal{O}_{d}$; see below, in Section 5 , some comments on the necessity of the hypothesis (1.10).

In the linear case $(F \equiv 0)$, the exact controllability to the trajectories is equivalent to the null controllability property. The following result holds:

Theorem 1.1. Let us assume that $F \equiv 0, \mathcal{O}_{d} \cap \mathcal{O} \neq \emptyset$ and the $\mu_{i}>0(i=1,2)$ are sufficiently large. Then, there exists a positive function $\hat{\rho}=\hat{\rho}(t)$ blowing up at $t=T$ with the following property: if $\bar{y}$ is the unique solution to (1.7) with $(F \equiv 0)$ associated to the initial state $\bar{y}^{0} \in L^{2}(\Omega)$ and the $y_{i, d}$ are such that

$$
\iint_{\mathcal{O}_{d} \times(0, T)} \hat{\rho}^{2}\left|\bar{y}-y_{i, d}\right|^{2} \mathrm{~d} x \mathrm{~d} t<+\infty, \quad i=1,2,
$$

for any $y^{0} \in L^{2}(\Omega)$, there exist controls $f \in L^{2}(\mathcal{O} \times(0, T))$ and associated Nash equilibria $\left(v^{1}, v^{2}\right)$ such that the corresponding solutions to (1.1) satisfy (1.9).

Roughly speaking, the assumption on the $\mu_{i}$ means that the followers must have moderate $L^{2}$ norms. On the other hand, the assumption (1.11) means that both $y_{1, d}$ and $y_{2, d}$ approach $\bar{y}$ as $t \rightarrow T$.

In the semilinear case, with $F$ being a locally Lipschitz-continuous function, we can consider the same controllability questions. However, it is important to note that, in this case, we lose the convexity of the functionals $J_{i}$ and the Nash equilibrium condition (1.4) is not necessarily equivalent to (1.5) and (1.6). For this reason, it is convenient to weaken the definition of equilibrium as follows:

Definition 1.2. Let $f \in L^{2}(\mathcal{O} \times(0, T))$ be given. The pair $\left(v^{1}, v^{2}\right)$ is called a Nash quasi-equilibrium of (1.1)-(1.2) associated to $f$ if the conditions (1.5) and (1.6) are satisfied.

For the semilinear case, we have the following result:

Theorem 1.3. Let us assume that $F \in W^{1, \infty}(\mathbb{R}), \mathcal{O}_{d} \cap \mathcal{O} \neq \emptyset$ and the $\mu_{i}>0(i=1,2)$ are sufficiently large. Let $\bar{y}$ be the unique solution to (1.7) associated to the initial state $\bar{y}^{0} \in L^{2}(\Omega)$ and let us assume that (1.11) holds, where $\hat{\rho}$ is the weight furnished by Theorem 1.1. Then, for each $y_{0} \in L^{2}(\Omega)$, there exist controls $f \in L^{2}(\mathcal{O} \times(0, T))$ and associated Nash quasi-equilibria $\left(v^{1}, v^{2}\right)$ such that the corresponding solutions to (1.1) satisfy (1.9).

A natural question is whether there are semilinear systems for which the concepts of Nash equilibrium and Nash quasi-equilibrium are equivalent. An answer is given by the following result:

Proposition 1.4. Let us assume that $F \in W^{2, \infty}(\mathbb{R})$ and $y_{i, d} \in L^{\infty}\left(\mathcal{O}_{i, d} \times(0, T)\right)(i=1,2)$. Suppose that $y_{0} \in$ $H_{0}^{1}(\Omega)$ (resp. $\left.y_{0} \in L^{2}(\Omega)\right)$ and $N \leq 14$ (resp. $\left.N \leq 12\right)$. Then, there exists $C>0$ such that, if $f \in L^{2}(\mathcal{O} \times(0, T)$ ) and the $\mu_{i}$ satisfy

$$
\mu_{i} \geq C\left(1+\|f\|_{L^{2}(\mathcal{O} \times(0, T))}\right),
$$

the conditions (1.4) and (1.5)-(1.6) are equivalent.

In this paper, we also analyze if a result like Theorem 1.1 holds true when the followers are constrained to belong to appropriate convex sets $\mathcal{U}_{i} \subset L^{2}\left(\mathcal{O}_{i} \times(0, T)\right)$. Thus, let $I_{1}$ and $I_{2}$ be two nonempty closed intervals with $0 \in I_{1} \cap I_{2}$, let us take

$$
\mathcal{U}_{i}=\left\{v \in L^{2}\left(\mathcal{O}_{i} \times(0, T)\right): v(x, t) \in I_{i} \quad \text { a.e. }\right\}, \quad i=1,2,
$$

and let us suppose that the minimization of $J_{1}$ and $J_{2}$ in (1.4) is subject to the restrictions $\hat{v}^{1} \in \mathcal{U}_{1}$ and $\hat{v}^{2} \in \mathcal{U}_{2}$. 
The controllability result is the following:

Theorem 1.5. Let us assume that $F \equiv 0, \mathcal{O}_{d} \cap \mathcal{O} \neq \emptyset$ and the $\mu_{i}>0(i=1,2)$ are sufficiently large. Let $\bar{y}$ be the unique solution to (1.7) associated to the initial state $\bar{y}^{0} \in L^{2}(\Omega)$. Then, for each $y_{0} \in L^{2}(\Omega)$, there exist controls $f \in L^{2}(\mathcal{O} \times(0, T))$ and associated Nash equilibria $\left(v^{1}, v^{2}\right) \in \mathcal{U}_{1} \times \mathcal{U}_{2}$ such that the corresponding solutions to (1.1) satisfy (1.9).

As mentioned above, the main novelty of this paper is that we deal with exact and not approximate controllability. There are other points that distinguish our contribution as well. Thus, contrarily to what was imposed in other previous papers (see for instance [11]), we do not make any assumption on the open sets $\mathcal{O}_{i}$. In particular, the $\mathcal{O}_{i}$ can be disjoint of $\mathcal{O}$, which is obviously the most interesting situation. On the other hand, the analysis and results also hold, after appropriate modifications, for $m$ followers with $m>2$.

The rest of the paper is organized as follows. In Section 2 we prove Theorem 1.1, which concerns the linear case. This result will be strongly used in the other sections. In Section 3 we prove Theorem 1.3 and Proposition 1.4. As a consequence, we see that the Stackelberg-Nash strategy can be applied to nonlinear problems and, also, that under adequate hypotheses on $F$, we still obtain a Nash equilibrium. Section 4 deals with the proof of Theorem 1.5. Finally, we present some additional comments and questions in Section 5.

\section{THE LINEAR CASE}

In this section we prove Theorem 1.1. The proof is long and, for clarity, has been decomposed in two parts. In Section 2.1 we will recall the existence, uniqueness and characterization of a Nash equilibrium (for fixed but arbitrary $f$ ); then, in Section 2.2, we will prove the desired controllability result.

Thanks to the linearity of the problem, we may reduce the exact controllability to the trajectories to a null controllability property. In fact, after the change of variable $y=z+\bar{y}$, it is immediate to see from (1.1) and (1.7), with $F \equiv 0$, that $z$ is the solution to the problem

$$
\left\{\begin{array}{lr}
z_{t}-\Delta z+a(x, t) z=f 1_{\mathcal{O}}+v^{1} 1_{\mathcal{O}_{1}}+v^{2} 1_{\mathcal{O}_{2}} & \text { in } Q \\
z=0 & \text { on } \Sigma \\
z(\cdot, 0)=z^{0} & \text { in } \Omega
\end{array}\right.
$$

where $z^{0}=y^{0}-\bar{y}^{0}$. It is clear that the condition (1.9) is equivalent to

$$
z(x, T)=0 \text { in } \Omega .
$$

Also, we can write the functionals $J_{i}$ in (1.2) in terms of $z$, which gives

$$
J_{i}\left(f ; v^{1}, v^{2}\right)=\frac{\alpha_{i}}{2} \iint_{\mathcal{O}_{i, d} \times(0, T)}\left|z-z_{i, d}\right|^{2} \mathrm{~d} x \mathrm{~d} t+\frac{\mu_{i}}{2} \iint_{\mathcal{O}_{i} \times(0, T)}\left|v^{i}\right|^{2} \mathrm{~d} x \mathrm{~d} t, \quad i=1,2,
$$

where $z_{i, d}:=y_{i, d}-\bar{y}(i=1,2)$.

\subsection{Nash equilibrium}

In this subsection, we will recall an existence/uniqueness result concerning a Nash equilibrium, in the sense of (1.4), for any $f \in L^{2}(\mathcal{O} \times(0, T))$. We will also recall a result which characterizes this Nash equilibrium in terms of the solution to an adjoint system. These results are due to Díaz and Lions (see $[3,4,15])$.

For the moment, we do not have to impose the assumption (1.10). This requirement only appears later, in Section 2.2, when the choice of $f$ has to be made. Accordingly, in this section we keep the notation $\mathcal{O}_{i, d}$ $(i=1,2)$. 


\subsubsection{Existence and uniqueness}

Let us introduce the spaces $\mathcal{H}_{i}:=L^{2}\left(\mathcal{O}_{i} \times(0, T)\right)$ and $\mathcal{H}:=\mathcal{H}_{1} \times \mathcal{H}_{2}$ and let us consider the operators $L_{i} \in \mathcal{L}\left(\mathcal{H}_{i} ; L^{2}(Q)\right)$ with $L_{i} v^{i}=z^{i}$, where $z^{i}$ is the solution to the system

$$
\begin{cases}z_{t}^{i}-\Delta z^{i}+a(x, t) z=v^{i} 1_{\mathcal{O}_{i}} & \text { in } Q, \\ z^{i}=0 & \text { on } \Sigma, \\ z^{i}(\cdot, 0)=0 & \text { in } \Omega .\end{cases}
$$

By definition, for any control $f$, the pair $\left(v^{1}, v^{2}\right)$ is a Nash equilibrium if and only if it satisfies (1.5) and (1.6), that is to say,

$$
\alpha_{i} \iint_{\mathcal{O}_{i, d} \times(0, T)}\left(z-z_{i, d}\right) w^{i} \mathrm{~d} x \mathrm{~d} t+\mu_{i} \iint_{\mathcal{O}_{i} \times(0, T)} v^{i} \hat{v}^{i} \mathrm{~d} x \mathrm{~d} t=0, \quad \forall \hat{v}^{i} \in \mathcal{H}_{i}
$$

where $w^{i}$ is the derivative of $z$ with respect to $v^{i}$ in the direction $\hat{v}^{i}$. Note that

$$
\begin{cases}w_{t}^{i}-\Delta w^{i}+a(x, t) w^{i}=\hat{v}^{i} 1_{\mathcal{O}_{i}} & \text { in } Q, \\ w^{i}=0 & \text { on } \Sigma, \\ w^{i}(\cdot, 0)=0 & \text { in } \Omega .\end{cases}
$$

Consequently, $L_{i} \hat{v}^{i}=w^{i}$. We also have $z=L_{1} v^{1}+L_{2} v^{2}+u$, where

$$
\begin{cases}u_{t}-\Delta u+a(x, t) u=f 1_{\mathcal{O}} & \text { in } Q, \\ u=0 & \text { on } \Sigma, \\ u(\cdot, 0)=z^{0} & \text { in } \Omega .\end{cases}
$$

Therefore, we may rewrite (2.3) in the form

$$
\begin{aligned}
\alpha_{i} \iint_{\mathcal{O}_{i, d} \times(0, T)}\left(L_{1} v^{1}+L_{2} v^{2}-\left(z_{i, d}-u\right)\right) L_{i} \hat{v}^{i} \mathrm{~d} x \mathrm{~d} t \\
+\mu_{i} \iint_{\mathcal{O}_{i} \times(0, T)} v^{i} \hat{v}^{i} \mathrm{~d} x \mathrm{~d} t=0, \quad \forall \hat{v}^{i} \in \mathcal{H}_{i}
\end{aligned}
$$

or

$$
\iint_{\mathcal{O}_{i} \times(0, T)}\left(\alpha_{i} L_{i}^{*}\left(\left(L_{1} v^{1}+L_{2} v^{2}-\left(z_{i, d}-u\right)\right) 1_{\mathcal{O}_{i, d}}\right)+\mu_{i} v^{i}\right) \hat{v}^{i} \mathrm{~d} x \mathrm{~d} t=0, \quad \forall \hat{v}^{i} \in \mathcal{H}_{i},
$$

where $L_{i}^{*} \in \mathcal{L}\left(L^{2}(Q) ; \mathcal{H}_{i}\right)$ is the adjoint of $L_{i}$. In other words, $\left(v^{1}, v^{2}\right)$ is a Nash equilibrium if and only if

$$
\alpha_{i} L_{i}^{*}\left(\left(L_{1} v^{1}+L_{2} v^{2}\right) 1_{\mathcal{O}_{i, d}}\right)+\mu_{i} v^{i}=\alpha_{i} L_{i}^{*}\left(\left(z_{i, d}-u\right) 1_{\mathcal{O}_{i, d}}\right) \text { in } \mathcal{H}_{i}, \quad i=1,2 .
$$

Let us introduce the operator $\mathbb{L} \in \mathcal{L}(\mathcal{H} ; \mathcal{H})$, given by

$$
\mathbb{L}\left(v^{1}, v^{2}\right)=\left(\alpha_{1} L_{1}^{*}\left(\left(L_{1} v^{1}+L_{2} v^{2}\right) 1_{\mathcal{O}_{1, d}}\right)+\mu_{1} v^{1}, \alpha_{2} L_{2}^{*}\left(\left(L_{1} v^{1}+L_{2} v^{2}\right) 1_{\mathcal{O}_{2, d}}\right)+\mu_{2} v^{2}\right),
$$

for all $\left(v^{1}, v^{2}\right) \in \mathcal{H}$. Then, the task is to prove the existence and uniqueness of a solution for the equation

$$
\mathbb{L}\left(v^{1}, v^{2}\right)=\Psi, \quad\left(v^{1}, v^{2}\right) \in \mathcal{H},
$$

where

$$
\Psi=\left(\alpha_{1} L_{1}^{*}\left(\left(z_{1, d}-u\right) 1_{\mathcal{O}_{1, d}}\right), \alpha_{2} L_{2}^{*}\left(\left(z_{2, d}-u\right) 1_{\mathcal{O}_{2, d}}\right)\right)
$$


In this direction, the following holds:

Proposition 2.1. Let us assume that

$$
\alpha_{1}\left\|1_{\mathcal{O}_{1, d}} L_{2}\right\|_{(1)}<4 \mu_{2} \quad \text { and } \quad \alpha_{2}\left\|1_{\mathcal{O}_{2, d}} L_{1}\right\|_{(2)}<4 \mu_{1},
$$

where $\|\cdot\|_{(i)}$ denotes the norm in the space $\mathcal{L}\left(\mathcal{H}_{3-i} ; L^{2}\left(\mathcal{O}_{i, d} \times(0, T)\right)\right)$. Then $\mathbb{L}$ is an isomorphism. In particular, for each $f \in L^{2}(\mathcal{O} \times(0, T))$, there exists exactly one Nash equilibrium $\left(v^{1}(f), v^{2}(f)\right)$ in the sense of (1.4).

Proof. From (2.4) and Young's inequality, we observe that

$$
\begin{aligned}
\left(\mathbb{L}\left(v^{1}, v^{2}\right),\left(v^{1}, v^{2}\right)\right)_{\mathcal{H}}= & \sum_{i=1}^{2} \mu_{i}\left\|v^{i}\right\|_{\mathcal{H}_{i}}^{2}+\sum_{i, j=1}^{2} \alpha_{i}\left(L_{j} v^{j}, L_{i} v^{i}\right)_{L^{2}\left(\mathcal{O}_{i, d} \times(0, T)\right)} \\
\geq & \sum_{i=1}^{2}\left(\mu_{i}\left\|v^{i}\right\|_{\mathcal{H}_{i}}^{2}+\alpha_{i}\left\|L_{i} v^{i}\right\|_{L^{2}\left(\mathcal{O}_{i, d} \times(0, T)\right)}^{2}\right) \\
& -\sum_{i=1}^{2} \alpha_{i}\left(\left\|L_{i} v^{i}\right\|_{L^{2}\left(\mathcal{O}_{i, d} \times(0, T)\right)}^{2}+\frac{1}{4}\left\|L_{3-i} v^{3-i}\right\|_{L^{2}\left(\mathcal{O}_{i, d} \times(0, T)\right)}^{2}\right) \\
\geq & \sum_{i=1}^{2}\left(\mu_{i}-\frac{\alpha_{3-i}}{4}\left\|1_{\mathcal{O}_{3-i, d}} L_{i}\right\|_{(3-i)}^{2}\right)\left\|v^{i}\right\|_{\mathcal{H}_{i}}^{2} .
\end{aligned}
$$

Therefore,

$$
\left(\mathbb{L}\left(\left(v^{1}, v^{2}\right),\left(v^{1}, v^{2}\right)\right)\right)_{\mathcal{H}} \geq \gamma\left\|\left(v^{1}, v^{2}\right)\right\|_{\mathcal{H}}^{2}, \quad \forall\left(v^{1}, v^{2}\right) \in \mathcal{H},
$$

where $\gamma=\min _{i}\left\{\mu_{i}-\alpha_{3-i}\left\|1_{\mathcal{O}_{3-i, d}} L_{i}\right\|_{(3-i)}^{2}\right\}>0$, see $(2.7)$.

Now, let us introduce the bilinear form $a: \mathcal{H} \times \mathcal{H} \rightarrow \mathbb{R}$, with

$$
a\left(\left(v^{1}, v^{2}\right),\left(\hat{v}^{1}, \hat{v}^{2}\right)\right):=\left(\mathbb{L}\left(\left(v^{1}, v^{2}\right),\left(\hat{v}^{1}, \hat{v}^{2}\right)\right)\right)_{\mathcal{H}} .
$$

From the definition of the operator $\mathbb{L}$ and the inequality $(2.8)$, we readily see that $a(\cdot, \cdot)$ is continuous and coercive on $\mathcal{H}$. Consequently, the Lax-Milgram's Theorem implies that, for any $\Phi \in \mathcal{H}^{\prime}$, there exists exactly one $\left(v^{1}, v^{2}\right) \in \mathcal{H}$ satisfying

$$
a\left(\left(v^{1}, v^{2}\right),\left(\hat{v}^{1}, \hat{v}^{2}\right)\right)=\left\langle\Phi,\left(\hat{v}^{1}, \hat{v}^{2}\right)\right\rangle_{\mathcal{H}^{\prime} \times \mathcal{H}} \quad \forall\left(\hat{v}^{1}, \hat{v}^{2}\right) \in \mathcal{H} ; \quad\left(v^{1}, v^{2}\right) \in \mathcal{H} .
$$

In particular, we get $(2.5)$ and the proof is done.

From the proof, it becomes clear that, under the assumptions of Proposition 2.1, for any $f \in L^{2}(\mathcal{O} \times(0, T))$ the associated Nash equilibrium $\left(v^{1}(f), v^{2}(f)\right)$ satisfies

$$
\left\|\left(v^{1}(f), v^{2}(f)\right)\right\|_{\mathcal{H}} \leq C\left(1+\|f\|_{L^{2}(\mathcal{O} \times(0, T))}\right),
$$

where the constant $C$ depends on $\Omega, \mathcal{O}, T, \mathcal{O}_{i}, \mathcal{O}_{i, d}, \alpha_{i}, \mu_{i},\left\|z_{0}\right\|$ and $\|a\|_{L^{\infty}(Q)}$. These estimates will be used below. Notice that, in view of $(2.9)$, the state $z$ associated to $f$ and $\left(v^{1}(f), v^{2}(f)\right)$ satisfies

$$
\|z\|_{L^{2}\left(0, T ; H_{0}^{1}(\Omega)\right)}+\left\|z_{t}\right\|_{L^{2}\left(0, T ; H^{-1}(\Omega)\right)} \leq C\left(1+\|f\|_{L^{2}(\mathcal{O} \times(0, T))}\right),
$$

where $C$ is as above. 


\subsubsection{Characterization of the Nash equilibrium}

We will express the followers $v^{1}(f)$ and $v^{2}(f)$ in terms of a new adjoint variable.

Let $f \in L^{2}(\mathcal{O} \times(0, T))$ be given. For any $\left(v^{1}, v^{2}\right) \in \mathcal{H}$, let us consider the associated state $z$ (the solution for $(2.1))$. In view of (2.3), it is very natural to introduce the adjoint states $\phi^{i}(i=1,2)$, with

$$
\begin{cases}-\phi_{t}^{i}-\Delta \phi^{i}+a(x, t) \phi^{i}=\alpha_{i}\left(z-z_{i, d}\right) 1_{\mathcal{O}_{i, d}} & \text { in } Q \\ \phi^{i}=0 & \text { on } \Sigma, \\ \phi^{i}(\cdot, T)=0 & \text { in } \Omega .\end{cases}
$$

Using integration by parts, we see that $\left(v^{1}, v^{2}\right)$ is a Nash equilibrium if and only if

$$
\iint_{\mathcal{O}_{i} \times(0, T)}\left(\phi^{i}+\mu_{i} v^{i}\right) \hat{v}^{i} \mathrm{~d} x \mathrm{~d} t=0, \quad \forall \hat{v}^{i} \in \mathcal{H}_{i}, \quad v^{i} \in \mathcal{H}_{i} .
$$

This directly implies that

$$
v^{i}=-\left.\frac{1}{\mu_{i}} \phi^{i}\right|_{\mathcal{O}_{i} \times(0, T)}, \quad i=1,2 .
$$

Let us gather all these informations in the same system. We obtain the following:

$$
\begin{cases}z_{t}-\Delta z+a(x, t) z=f 1_{\mathcal{O}}-\sum_{i=1}^{2} \frac{1}{\mu_{i}} \phi^{i} 1_{\mathcal{O}_{i}} & \text { in } Q, \\ -\phi_{t}^{i}-\Delta \phi^{i}+a(x, t) \phi^{i}=\alpha_{i}\left(z-z_{i, d}\right) 1_{\mathcal{O}_{i, d}} & \text { in } Q, \\ z=0, \quad \phi^{i}=0 & \text { on } \Sigma, \\ z(\cdot, 0)=z^{0}, \quad \phi^{i}(\cdot, T)=0 & \text { in } \Omega .\end{cases}
$$

Recall that our main objective is to prove the null controllability of $z$ at time $t=T$. Therefore, the task is to find a distributed control $f \in L^{2}(\mathcal{O} \times(0, T))$ such that the solution to (2.11) satisfies (2.2).

\subsection{Null controllability}

In this subsection, we will achieve the proof of Theorem 1.1.

We will establish an observability inequality for the system

$$
\begin{cases}-\psi_{t}-\Delta \psi+a(x, t) \psi=\sum_{i=1}^{2} \alpha_{i} \gamma^{i} 1_{\mathcal{O}_{i, d}} & \text { in } Q, \\ \gamma_{t}^{i}-\Delta \gamma^{i}+a(x, t) \gamma^{i}=-\frac{1}{\mu_{i}} \psi 1_{\mathcal{O}_{i}} & \text { in } Q, \\ \psi=0, \quad \gamma^{i}=0 & \text { on } \Sigma, \\ \psi(\cdot, T)=\psi^{T}, \quad \gamma^{i}(\cdot, 0)=0 & \text { in } \Omega,\end{cases}
$$

which can be viewed as the adjoint of (2.11). This will suffice. This observability estimate is given in the following result:

Proposition 2.2. Assume that (1.10) holds, $\mathcal{O}_{d} \cap \mathcal{O} \neq \emptyset$ and the $\mu_{i}$ are sufficiently large. There exist $C>0$, only depending on $\Omega, \mathcal{O}, T, \mathcal{O}_{i}, \mathcal{O}_{d}, \alpha_{i}, \mu_{i}$ and $\|a\|_{L^{\infty}(Q)}$ and a weight function $\hat{\rho}=\hat{\rho}(t)$ blowing up at $t=T$, only depending on $\Omega, \mathcal{O}, \mathcal{O}_{d}, T$ and $\|a\|_{L^{\infty}(Q)}$, such that, for any $\psi^{T} \in L^{2}(\Omega)$, the following inequality holds true for the solution $\left(\psi, \gamma^{i}\right)$ of $(2.12)$ :

$$
\int_{\Omega}|\psi(x, 0)|^{2} \mathrm{~d} x+\sum_{i=1}^{2} \iint_{Q} \hat{\rho}^{-2}\left|\gamma^{i}\right|^{2} \mathrm{~d} x \mathrm{~d} t \leq C \iint_{\mathcal{O} \times(0, T)}|\psi|^{2} \mathrm{~d} x \mathrm{~d} t .
$$


Let us assume for a moment that Proposition 2.2 holds and let us prove the controllability result in Theorem 1.1. From a well known duality argument, we have that, for any $z^{0} \in L^{2}(\Omega)$ and any $\psi^{T} \in L^{2}(\Omega)$,

$$
\int_{\Omega}\left[z(x, T) \psi^{T}(x)-z^{0}(x) \psi(x, 0)\right] \mathrm{d} x=\iint_{\mathcal{O} \times(0, T)} f \psi \mathrm{d} x \mathrm{~d} t-\sum_{i=1}^{2} \alpha_{i} \iint_{\mathcal{O}_{d} \times(0, T)} z_{i, d} \gamma^{i} \mathrm{~d} x \mathrm{~d} t,
$$

where $\left(z, \phi^{1}, \phi^{2}\right)$ and $\left(\psi, \gamma^{1}, \gamma^{2}\right)$ are the solutions to (2.11) and (2.12), respectively associated to $z^{0}$ and $\psi^{T}$. Thus, to prove the null controllability property is equivalent to find, for each $z^{0} \in L^{2}(\Omega)$, a control $f$ such that, for any $\psi^{T} \in L^{2}(\Omega)$, one has

$$
\iint_{\mathcal{O} \times(0, T)} f \psi \mathrm{d} x \mathrm{~d} t=-\int_{\Omega} z^{0}(x) \psi(x, 0) \mathrm{d} x+\sum_{i=1}^{2} \alpha_{i} \iint_{\mathcal{O}_{d} \times(0, T)} z_{i, d} \gamma^{i} \mathrm{~d} x \mathrm{~d} t .
$$

There are several ways to show that (2.13) implies the existence of such a control. They rely on well known arguments. For completeness, let us sketch one of them.

For each $\epsilon>0$, let us consider the following functional:

$$
\begin{aligned}
F_{\epsilon}\left(\psi^{T}\right):= & \frac{1}{2} \iint_{\mathcal{O} \times(0, T)}|\psi|^{2} \mathrm{~d} x \mathrm{~d} t+\epsilon\left\|\psi^{T}\right\|+\int_{\Omega} z^{0}(x) \psi(x, 0) \mathrm{d} x \\
& -\sum_{i=1}^{2} \alpha_{i} \iint_{\mathcal{O}_{d} \times(0, T)} z_{i, d} \gamma^{i} \mathrm{~d} x \mathrm{~d} t, \quad \forall \psi^{T} \in L^{2}(\Omega) .
\end{aligned}
$$

It is then clear that $F_{\epsilon}: L^{2}(\Omega) \rightarrow \mathbb{R}$ is continuous and strictly convex. Moreover,

$$
\begin{aligned}
F_{\epsilon}\left(\psi^{T}\right) \geq & \frac{1}{4} \iint_{\mathcal{O} \times(0, T)}|\psi|^{2} \mathrm{~d} x \mathrm{~d} t \\
& -C\left(\int_{\Omega}\left|z^{0}\right|^{2} \mathrm{~d} x+\sum_{i=1}^{2} \alpha_{i}^{2} \iint_{\mathcal{O}_{d} \times(0, T)} \hat{\rho}^{2}\left|z_{i, d}\right|^{2} \mathrm{~d} x \mathrm{~d} t\right) \\
& +\epsilon\left\|\psi^{T}\right\|,
\end{aligned}
$$

where $C$ and $\hat{\rho}$ are furnished by Proposition 2.2. Consequently, $F_{\epsilon}$ is also coercive in $L^{2}(\Omega)$. Note that, here, we have used the assumption (1.11) on $z_{i, d}=y_{i, d}-\bar{y}$.

Let $\psi_{\epsilon}^{T}$ be the unique minimizer of $F_{\epsilon}$. Then, either $\psi_{\epsilon}^{T}=0$ or

$$
\left\langle F_{\epsilon}^{\prime}\left(\psi_{\epsilon}^{T}\right), \psi^{T}\right\rangle=0, \quad \forall \psi^{T} \in L^{2}(\Omega)
$$

Suppose that $\psi_{\epsilon}^{T} \neq 0$. In this case, we have

$$
\begin{gathered}
\iint_{\mathcal{O} \times(0, T)} \psi_{\epsilon} \psi \mathrm{d} x \mathrm{~d} t+\epsilon\left(\frac{\psi_{\epsilon}^{T}}{\left\|\psi_{\epsilon}^{T}\right\|}, \psi^{T}\right)+\int_{\Omega} z^{0}(x) \psi(x, 0) \mathrm{d} x \\
-\sum_{i=1}^{2} \alpha_{i} \iint_{\mathcal{O}_{d} \times(0, T)} z_{i, d} \gamma^{i} \mathrm{~d} x \mathrm{~d} t=0, \quad \forall \psi^{T} \in L^{2}(\Omega),
\end{gathered}
$$

where we have denoted by $\left(\psi_{\epsilon}, \gamma_{\epsilon}^{1}, \gamma_{\epsilon}^{2}\right)$ the solution to $(2.12)$ corresponding to $\psi^{T}=\psi_{\epsilon}^{T}$. Taking $f=f_{\epsilon}:=$ $\psi_{\epsilon} 1_{\mathcal{O} \times(0, T)}$ in (2.14), denoting by $z_{\epsilon}$ the associated state and comparing to $(2.15)$, we see that

$$
\int_{\Omega}\left(z_{\epsilon}(x, T)-\frac{\epsilon}{\left\|\psi_{\epsilon}^{T}\right\|} \psi_{\epsilon}^{T}\right) \psi^{T}(x) \mathrm{d} x=0, \quad \forall \psi^{T} \in L^{2}(\Omega)
$$


which implies

$$
\left\|z_{\epsilon}(\cdot, T)\right\| \leq \epsilon .
$$

On the other hand, from (2.13) and (2.15) we also have

$$
\left\|f_{\epsilon}\right\|_{L^{2}(\mathcal{O} \times(0, T))} \leq C\left(\int_{\Omega}\left|z^{0}\right|^{2} \mathrm{~d} x+\sum_{i=1}^{2} \iint_{\mathcal{O}_{d} \times(0, T)} \hat{\rho}^{2}\left|z_{i, d}\right|^{2} \mathrm{~d} x \mathrm{~d} t\right)^{1 / 2},
$$

that is, $f_{\epsilon}$ is uniformly bounded in $L^{2}(\mathcal{O} \times(0, T))$.

Obviously, we also have (2.16) and (2.17) when $\psi_{\epsilon}^{T}=0$ and we take $f_{\epsilon}=0$.

In view of these inequalities, we can easily deduce a uniform estimate for $z_{\epsilon}$. Then, taking limits as $\epsilon \rightarrow 0$, we conclude that null controllability holds. This ends the proof of Theorem 1.1.

Remark 2.3. The leader control we have constructed is the unique solution to the extremal problem (1.8)-(1.9). This claim can be justified as follows:

1. For each $\epsilon>0$, there exists exactly one minimal $L^{2}$ norm control $f_{\epsilon}$ such that the associated state, i.e. the corresponding solution to (2.11), satisfies (2.16).

2. From the weak lower semicontinuity of the terms in $F_{\epsilon}$, it is clear that any weak limit of a subsequence of $\left\{f_{\epsilon}\right\}$ minimizes the $L^{2}$ norm in the family of the null controls for $z$. Consequently, this is the case for $f$.

Now, we will give the proof of Proposition 2.2.

Proof of Proposition 2.2. The assumption (1.10) will be used here.

Since $\mathcal{O}_{d} \cap \mathcal{O} \neq \emptyset$, there exists a non-empty open set $\omega$ satisfying $\omega \subset \subset \mathcal{O}_{d} \cap \mathcal{O}$. Let $\eta_{0}=\eta_{0}(x)$ be a function satisfying

$$
\left\{\begin{array}{l}
\eta_{0} \in C^{2}(\bar{\Omega}), \eta_{0}>0 \text { in } \Omega, \eta_{0}=0 \text { on } \Gamma \\
\left|\nabla \eta_{0}\right|>0 \text { in } \bar{\Omega} \backslash \omega
\end{array}\right.
$$

Such a function $\eta_{0}$ always exists (see [7]).

Let us introduce the weight functions

$$
\sigma(x, t):=\frac{\left.\mathrm{e}^{4 \lambda\left\|\eta^{0}\right\|_{L} \infty(\Omega)}-\mathrm{e}^{\lambda\left(2\left\|\eta^{0}\right\|_{L} \infty(\Omega)\right.}+\eta^{0}(x)\right)}{t(T-t)}, \quad \xi(x, t):=\frac{\left.\mathrm{e}^{\lambda\left(2\left\|\eta^{0}\right\|_{L} \infty(\Omega)\right.}+\eta^{0}(x)\right)}{t(T-t)}
$$

and the notation

$$
\begin{aligned}
I_{m}(\psi):= & s^{m-4} \lambda^{m-3} \iint_{Q} \mathrm{e}^{-2 s \sigma} \xi^{m-4}\left(\left|\psi_{t}\right|^{2}+|\Delta \psi|^{2}\right) \mathrm{d} x \mathrm{~d} t \\
& +s^{m-2} \lambda^{m-1} \iint_{Q} \mathrm{e}^{-2 s \sigma} \xi^{m-2}|\nabla \psi|^{2} \mathrm{~d} x \mathrm{~d} t \\
& +s^{m} \lambda^{m+1} \iint_{Q} \mathrm{e}^{-2 s \sigma} \xi^{m}|\psi|^{2} \mathrm{~d} x \mathrm{~d} t .
\end{aligned}
$$

From the usual Carleman inequalities (see $[5,7,13]$ ), we have:

$$
\begin{aligned}
I_{3}(\psi) \leq & C\left(\iint_{Q} \mathrm{e}^{-2 s \sigma}\left|\alpha_{1} \gamma^{1} 1_{\mathcal{O}_{1, d}}+\alpha_{2} \gamma^{2} 1_{\mathcal{O}_{2, d}}\right|^{2} \mathrm{~d} x \mathrm{~d} t\right. \\
& \left.+s^{3} \lambda^{4} \iint_{\omega \times(0, T)} \mathrm{e}^{-2 s \sigma} \xi^{3}|\psi|^{2} \mathrm{~d} x \mathrm{~d} t\right) .
\end{aligned}
$$


Since (1.10) holds, we introduce $h:=\alpha_{1} \gamma^{1}+\alpha_{2} \gamma^{2}$ and we obtain

$$
\begin{aligned}
\iint_{Q} \mathrm{e}^{-2 s \sigma}|h|^{2} \mathrm{~d} x \mathrm{~d} t & \leq I_{0}(h) \\
& \leq C\left(s^{-3} \lambda^{-3} \iint_{Q} \mathrm{e}^{-2 s \sigma} \xi^{-3}|\psi|^{2} \mathrm{~d} x \mathrm{~d} t+\lambda \iint_{\omega \times(0, T)} \mathrm{e}^{-2 s \sigma}|h|^{2} \mathrm{~d} x \mathrm{~d} t\right),
\end{aligned}
$$

for all large $s$ and $\lambda$ and some $C$ only depending on $\Omega, \omega$ and $T$.

We can observe that, in $\omega \times(0, T)$, one has $h=-\psi_{t}-\Delta \psi+a \psi$. Consequently, by introducing an appropriate cut-off function $\zeta$ and integrating by parts, we get

$$
\begin{aligned}
\lambda \iint_{\omega \times(0, T)} \mathrm{e}^{-2 s \sigma}|h|^{2} \mathrm{~d} x \mathrm{~d} t & \leq \lambda \iint_{\omega^{\prime} \times(0, T)} \zeta \mathrm{e}^{-2 s \sigma} h\left(-\psi_{t}-\Delta \psi+a \psi\right) \mathrm{d} x \mathrm{~d} t \\
& \leq \epsilon I_{0}(h)+C_{\epsilon} s^{4} \lambda^{5} \iint_{\omega^{\prime} \times(0, T)} \xi^{4} \mathrm{e}^{-2 s \sigma}|\psi|^{2} \mathrm{~d} x \mathrm{~d} t
\end{aligned}
$$

where $\omega^{\prime}$ is a new open set satisfying $\omega \subset \omega^{\prime} \subset \mathcal{O}_{d} \cap \mathcal{O}$. From (2.20)-(2.22), we find that, for some $C>0$,

$$
I_{3}(\psi)+I_{0}(h) \leq C \iint_{\omega^{\prime} \times(0, T)} \xi^{4} \mathrm{e}^{-2 s \sigma}|\psi|^{2} \mathrm{~d} x \mathrm{~d} t .
$$

Now, let us introduce $\ell=\ell(t)$, with

$$
\ell(t):= \begin{cases}T^{2} / 4 & \text { for } 0 \leq t \leq T / 2 \\ t(T-t) & \text { for } T / 2 \leq t \leq T\end{cases}
$$

and the functions

$$
\bar{\sigma}(x, t):=\frac{\left.\mathrm{e}^{4 \lambda\left\|\eta^{0}\right\|_{L \infty}(\Omega)}-\mathrm{e}^{\lambda\left(2\left\|\eta^{0}\right\|_{L} \infty(\Omega)\right.}+\eta^{0}(x)\right)}{\ell(t)}, \quad \bar{\xi}(x, t):=\frac{\left.\mathrm{e}^{\lambda\left(2\left\|\eta^{0}\right\|_{L} \infty(\Omega)\right.}+\eta^{0}(x)\right)}{\ell(t)} .
$$

Let us denote by $\bar{I}_{m}(\psi)$ the right-hand side of (2.19) with $\sigma$ and $\xi$ repectively replaced by $\bar{\sigma}$ and $\bar{\xi}$. Then, arguing as in ([6], Lem. 1), it is not difficult to see from (2.23) and the PDEs satisfied by $\psi$ and the $\gamma^{i}$ that there exists a constant $C>0$, such that

$$
\|\psi(\cdot, 0)\|^{2}+\bar{I}_{3}(\psi)+\bar{I}_{0}(h) \leq C \iint_{\omega^{\prime} \times(0, T)} \xi^{4} \mathrm{e}^{-2 s \bar{\sigma}}|\psi|^{2} \mathrm{~d} x \mathrm{~d} t .
$$

Let us introduce

$$
\bar{\sigma}^{*}(t):=\max _{x \in \bar{\Omega}} \bar{\sigma}(x, t), \quad \hat{\rho}(t):=\mathrm{e}^{s \bar{\sigma}^{*}(t)} .
$$

Then $\hat{\rho}=\hat{\rho}(t)$ is a positive nondecreasing function in $C^{1}([0, T])$ that blows up at $t=T$. Observe that $\hat{\rho}$ is determined by the Carleman weight $\mathrm{e}^{s \sigma}$, that depends on $\Omega, \mathcal{O}, \mathcal{O}_{d}, T$ and $\|a\|_{L^{\infty}(Q)}$, but can be chosen independently of $\mathcal{O}_{i}, \alpha_{i}$ and $\mu_{i}$.

Using again the PDEs satisfied by the $\gamma^{i}$ in (2.12), we readily see that

$$
\begin{aligned}
\frac{1}{2} \frac{d}{\mathrm{~d} t} \int_{\Omega} \hat{\rho}^{-2}\left|\gamma^{i}\right|^{2} \mathrm{~d} x+\int_{\Omega} \hat{\rho}^{-2}\left|\nabla \gamma^{i}\right|^{2} \mathrm{~d} x & =-\frac{1}{\mu_{i}} \int_{\mathcal{O}_{i}} \hat{\rho}^{-2} \psi \gamma^{i} \mathrm{~d} x-\int_{\Omega} \hat{\rho}^{-3} \hat{\rho}_{t}\left|\gamma^{i}\right|^{2} \mathrm{~d} x-\int_{\Omega} \hat{\rho}^{-2} a\left|\gamma^{i}\right|^{2} \mathrm{~d} x \\
& \leq \frac{1}{\mu_{i}^{2}} \int_{\mathcal{O}_{i}} \hat{\rho}^{-2}|\psi|^{2} \mathrm{~d} x+\left(1+\|a\|_{L^{\infty}(Q)}\right) \int_{\Omega} \hat{\rho}^{-2}\left|\gamma^{i}\right|^{2} \mathrm{~d} x .
\end{aligned}
$$


Thus, from Gronwall's Lemma and the fact that $\gamma^{i}(x, 0) \equiv 0$, it follows that

$$
\left(\int_{\Omega} \hat{\rho}^{-2}\left|\gamma^{i}\right|^{2} \mathrm{~d} x\right)(\tau) \leq C \iint_{\mathcal{O}_{i} \times(0, T)} \hat{\rho}^{-2}|\psi|^{2} \mathrm{~d} x \mathrm{~d} t, \quad \forall \tau \in[0, T] .
$$

Since the right-hand side of $(2.26)$ is bounded up to a multiplicative constant by $\bar{I}_{3}(\psi)$, in view of $(2.25)$ and (2.26), we find that

$$
\|\psi(\cdot, 0)\|^{2}+\sum_{i=1}^{2} \iint_{Q} \hat{\rho}^{-2}\left|\gamma^{i}\right|^{2} \mathrm{~d} x \mathrm{~d} t \leq C \iint_{\mathcal{O} \times(0, T)} \xi^{4} \mathrm{e}^{-2 s \bar{\sigma}}|\psi|^{2} \mathrm{~d} x \mathrm{~d} t,
$$

which implies (2.13). This ends the proof.

Remark 2.4. If, instead of (1.10), we assume that the main hypothesis in [11], namely

$$
\mathcal{O}_{i} \subset \mathcal{O}, \quad i=1,2,
$$

is satisfied, the same result holds. Indeed, multiplying the second PDE in $(2.12)$ by $\gamma^{i}$ and integrating $\Omega \times(0, t)$, we get

$$
\left\|\gamma^{i}(\cdot, t)\right\|^{2} \leq C \int_{0}^{t}\left\|\gamma^{i}(\cdot, s)\right\|^{2} \mathrm{~d} s+\frac{C}{\mu_{i}^{2}} \iint_{\mathcal{O}_{i} \times(0, t)}|\psi(x, s)|^{2} \mathrm{~d} x \mathrm{~d} s, \quad \forall t \in[0, T] .
$$

Consequently, by Gronwall's Lemma and (2.28), it follows that

$$
\left\|\gamma^{i}(\cdot, t)\right\|^{2} \leq C \iint_{\mathcal{O}_{i} \times(0, t)}|\psi(x, s)|^{2} \mathrm{~d} x \mathrm{~d} s \leq C \iint_{\mathcal{O} \times(0, t)}|\psi(x, s)|^{2} \mathrm{~d} x \mathrm{~d} s, \quad \forall t \in[0, T] .
$$

By replacing this inequality in the first term on the right-hand side of (2.20) and arguing again as in ([6], Lem. 1) we obtain

$$
\|\psi(\cdot, 0)\|^{2}+\bar{I}_{3}(\psi) \leq C \iint_{\mathcal{O} \times(0, T)} \xi^{4} \mathrm{e}^{-2 s \bar{\sigma}}|\psi|^{2} \mathrm{~d} x \mathrm{~d} t .
$$

This last inequality, together with (2.26), gives (2.27).

\section{ThE SEMiLineAR CASE}

In this section, we will analyze the controllability of a more general model, with a not necessarily vanishing function F. Our goals are to prove Theorem 1.3 and Proposition 1.4.

\subsection{Characterization of Nash quasi-equilibria}

As already mentioned in Section 1, in the semilinear case, the convexity of the functionals $J_{i}$ is not guaranteed. Consequently, it is not clear whether the definition of Nash equilibria used in the linear case is the good one. For this reason, we must re-define the concept of Nash optimality (recall Def. 1.2).

Notice that (1.5)-(1.6) is equivalent to

$$
\left\{\begin{array}{l}
\alpha_{i} \iint_{\mathcal{O}_{d} \times(0, T)}\left(y-y_{i, d}\right) p^{i} \mathrm{~d} x \mathrm{~d} t+\mu_{i} \iint_{\mathcal{O}_{i} \times(0, T)} v^{i} \hat{v}^{i} \mathrm{~d} x \mathrm{~d} t=0 \\
\forall \hat{v}^{i} \in \mathcal{H}_{i}, \quad v^{i} \in \mathcal{H}_{i}, \quad i=1,2,
\end{array}\right.
$$

where we have denoted by $p^{i}$ the derivative of the state $y$ with respect to $v^{i}$ in the direction $\hat{v}^{i}$. Obviously, one has

$$
\begin{cases}p_{t}^{i}-\Delta p^{i}+a(x, t) p^{i}=F^{\prime}(y) p^{i}+\hat{v}^{i} 1_{\mathcal{O}_{i}} & \text { in } Q, \\ p^{i}=0 & \text { on } \Sigma, \\ p^{i}(\cdot, 0)=0 & \text { in } \Omega .\end{cases}
$$


Let us introduce the adjoint systems

$$
\begin{cases}-\phi_{t}^{i}-\Delta \phi^{i}+a(x, t) \phi^{i}=F^{\prime}(y) \phi^{i}+\alpha_{i}\left(y-y_{i, d}\right) 1_{\mathcal{O}_{d}} & \text { in } Q, \\ \phi^{i}=0 & \text { on } \Sigma, \\ \phi^{i}(\cdot, T)=0 & \text { in } \Omega .\end{cases}
$$

Then, a short computation shows that (3.1) can be written equivalently as follows:

$$
\iint_{\mathcal{O}_{i} \times(0, T)}\left(\phi^{i}+\mu_{i} v^{i}\right) \hat{v}^{i} \mathrm{~d} x \mathrm{~d} t=0, \quad \forall \hat{v}^{i} \in \mathcal{H}_{i}, \quad v^{i} \in \mathcal{H}_{i}, \quad i=1,2 .
$$

As a consequence, we get the following characterization of any Nash quasi-equilibrium:

$$
v^{i}=-\left.\frac{1}{\mu_{i}} \phi^{i}\right|_{\mathcal{O}_{i} \times(0, T)}, \quad i=1,2 .
$$

In this way, we have the following optimality system:

$$
\begin{cases}y_{t}-\Delta y+a(x, t) y=F(y)+f 1_{\mathcal{O}}-\frac{1}{\mu_{1}} \phi^{1} 1_{\mathcal{O}_{1}}-\frac{1}{\mu_{2}} \phi^{2} 1_{\mathcal{O}_{2}} \text { in } Q, \\ -\phi_{t}^{i}-\Delta \phi^{i}+a(x, t) \phi^{i}=F^{\prime}(y) \phi^{i}+\alpha_{i}\left(y-y_{i, d}\right) 1_{\mathcal{O}_{i, d}} & \text { in } Q, \\ y=0, \quad \phi^{i}=0 & \text { on } \Sigma, \\ y(\cdot, 0)=y^{0}, \quad \phi^{i}(\cdot, T)=0 & \text { in } \Omega .\end{cases}
$$

\subsection{Proof of Theorem 1.3}

The Proof of Theorem 1.3 follows some arguments that are nowadays standard and rely on the ideas introduced in $[7,23]$. It is divided in three steps: first, we perform a change of variable that reduces the task to solve a null controllability problem; then, this is rewritten as a fixed-point equation in $L^{2}(Q)$; in particular, we use again Carleman inequalities and energy estimates to deduce an observability inequality for the adjoint of a linearized system; finally, in a third step, we use some compactness properties of the system and we prove the existence of a fixed-point.

Step 1. We must find a leader control $f \in L^{2}(\mathcal{O} \times(0, T))$ such that the solution $\left(y, \phi^{1}, \phi^{2}\right)$ to (3.3) satisfies (1.9). In fact, by introducing the change of variable $z=y-\bar{y}$, we can rewrite (3.3) in the form

$$
\begin{cases}z_{t}-\Delta z+a(x, t) z=G(x, t ; z) z+f 1_{\mathcal{O}}-\frac{1}{\mu_{1}} \phi^{1} 1_{\mathcal{O}_{1}}-\frac{1}{\mu_{2}} \phi^{2} 1_{\mathcal{O}_{2}} & \text { in } Q, \\ -\phi_{t}^{i}-\Delta \phi^{i}+a(x, t) \phi^{i}=F^{\prime}(z+\bar{y}) \phi^{i}+\alpha_{i}\left(z-z_{i, d}\right) 1_{\mathcal{O}_{d}} & \text { in } Q, \\ z=0, \quad \phi^{i}=0 & \text { on } \Sigma, \\ z(\cdot, 0)=z^{0}, \quad \phi^{i}(\cdot, T)=0 & \text { in } \Omega,\end{cases}
$$

where $z_{i, d}:=y_{i, d}-\bar{y}, z^{0}=y^{0}-\bar{y}(\cdot, 0)$ and

$$
G(x, t ; z)=\int_{0}^{1} F^{\prime}(\bar{y}(x, t)+\sigma z) \mathrm{d} \sigma .
$$

In this way, obviously, what we have to prove is the null controllability for $z$ in (3.4).

Step 2. For each $z \in L^{2}(Q)$ and each $f \in L^{2}(\mathcal{O} \times(0, T))$, let us introduce the linear system

$$
\begin{cases}w_{t}-\Delta w+a(x, t) w=G(x, t ; z) w+f 1_{\mathcal{O}}-\frac{1}{\mu_{1}} \phi^{1} 1_{\mathcal{O}_{1}}-\frac{1}{\mu_{2}} \phi^{2} 1_{\mathcal{O}_{2}} & \text { in } Q, \\ -\phi_{t}^{i}-\Delta \phi^{i}+a(x, t) \phi^{i}=F^{\prime}(z+\bar{y}) \phi^{i}+\alpha_{i}\left(w-z_{i, d}\right) 1_{\mathcal{O}_{d}} & \text { in } Q, \\ w=0, \quad \phi^{i}=0 & \text { on } \Sigma, \\ w(\cdot, 0)=z^{0}, \quad \phi^{i}(\cdot, T)=0 . & \text { in } \Omega .\end{cases}
$$


By hypothesis, there exists $K>0$ such that

$$
|G(x, t ; s)|+\left|F^{\prime}(s)\right| \leq K, \quad \forall(x, t, s) \in Q \times \mathbb{R} .
$$

Note that, arguing as in Section 2.1, it can be proved that, if $\mu_{1}$ and $\mu_{2}$ are sufficiently large, (3.5) possesses exactly one solution for each $f \in L^{2}(\mathcal{O} \times(0, T))$. Furthermore, one has

$$
\|w\|_{L^{2}\left(0, T ; H_{0}^{1}(\Omega)\right)}+\left\|w_{t}\right\|_{L^{2}\left(0, T ; H^{-1}(\Omega)\right)} \leq C\left(1+\|f\|_{L^{2}(\mathcal{O} \times(0, T))}\right),
$$

where $C$ depends on $\Omega, \mathcal{O}, T, \mathcal{O}_{d}, \alpha_{i}, \mu_{i}, K,\|a\|_{L^{\infty}(Q)}$ and $\left\|z_{0}\right\|$.

Let us introduce the mapping $\Lambda: L^{2}(Q) \rightarrow L^{2}(Q)$, with $\Lambda(z)=w_{z}$, for all $z \in L^{2}(Q)$, where $w_{z}$ is the state associated to the minimal $L^{2}$ norm null control $f_{z}$ for the linear system (3.5). In other words, $w_{z}$ is, together with $\phi_{z}^{1}, \phi_{z}^{2}$ and $f_{z}$, the unique solution to (3.5) and $f_{z}$ minimizes (1.3) subject to the constraint

$$
w(\cdot, T)=0 \quad \text { in } \quad \Omega .
$$

The goal is now to prove the null controllability of (3.5). To this purpose, we will make use again of a suitable global Carleman inequality for the solutions to the adjoint system, that is,

$$
\left\{\begin{array}{lr}
-\psi_{z, t}-\Delta \psi_{z}+a(x, t) \psi_{z}=G(x, t ; z) \psi_{z}+\left(\alpha_{1} \gamma_{z}^{1}+\alpha_{2} \gamma_{z}^{2}\right) 1_{\mathcal{O}_{d}} & \text { in } Q, \\
\gamma_{z, t}^{i}-\Delta \gamma_{z}^{i}=F^{\prime}(z+\bar{y}) \gamma_{z}^{i}-\frac{1}{\mu_{i}} \psi_{z} 1_{\mathcal{O}_{i}} & \text { in } Q, \\
\psi_{z}=0, \quad \gamma_{z}^{i}=0 & \text { on } \Sigma, \\
\psi_{z}(\cdot, T)=\psi^{T}, \quad \gamma_{z}^{i}(\cdot, 0)=0 & \text { in } \Omega .
\end{array}\right.
$$

In the context of (3.7), we have the following:

Proposition 3.1. Assume that (1.10) holds, $\mathcal{O}_{d} \cap \mathcal{O} \neq \emptyset$ and the $\mu_{i}$ are sufficiently large. There exist a constant $C>0$, only depending on $\Omega, \mathcal{O}, T, \mathcal{O}_{i}, \mathcal{O}_{d}, \alpha_{i}, \mu_{i}, K$ and $\|a\|_{L^{\infty}(Q)}$ and a weight function $\hat{\rho}=\hat{\rho}(x, t)$, only depending on $\Omega, \mathcal{O}, \mathcal{O}_{d}, T, K$ and $\|a\|_{L^{\infty}(Q)}$ and blowing up at $t=T$, such that the following observability inequality holds true for any $\psi^{T} \in L^{2}(\Omega)$ and any $z \in L^{2}(Q)$ :

$$
\int_{\Omega}\left|\psi_{z}(x, 0)\right|^{2} \mathrm{~d} x+\sum_{i=1}^{2} \iint_{Q} \hat{\rho}^{-2}\left|\gamma_{z}^{i}\right|^{2} \mathrm{~d} x \mathrm{~d} t \leq C \iint_{\mathcal{O} \times(0, T)}\left|\psi_{z}\right|^{2} \mathrm{~d} x \mathrm{~d} t .
$$

The proof is almost identical to the proof of Proposition 2.2 and, for brevity, is omitted.

This result leads, as in Section 2.2, to the existence of a minimal norm null control $f_{z} \in L^{2}(\mathcal{O} \times(0, T))$ for (3.5). Furthermore it is clear that there exists a positive constant $C$, only depending on $\Omega, \mathcal{O}, T, \mathcal{O}_{i}, \mathcal{O}_{d}$, $\alpha_{i}, \mu_{i}, K,\|a\|_{L^{\infty}(Q)}$ and $\left\|z_{0}\right\|$, such that

$$
\left\|f_{z}\right\|_{L^{2}(\mathcal{O} \times(0, T))}^{2} \leq C, \quad \forall z \in L^{2}(Q) .
$$

This argument proves that $\Lambda$ is well defined.

Step 3. Taking into account (3.6) and (3.8), we see that $w_{z}$ is uniformly bounded in $L^{2}\left(0, T ; H_{0}^{1}(\Omega)\right)$ and $w_{z, t}$ is uniformly bounded in $L^{2}\left(0, T, H^{-1}(\Omega)\right)$. In view of the classical Aubin-Lions' Compactness Theorem, this means that $\Lambda$ maps the whole space $L^{2}(Q)$ into a compact set. On the other hand, the mapping $z \mapsto \Lambda(z)$ is obviously continuous. Therefore, we can use Schauder's Fixed-Point Theorem to ensure the semilinear controllability result. This ends the Proof of Theorem 1.3. 


\subsection{Equilibria and quasi-equilibria}

The aim of this subsection is to prove Proposition 1.4, that is, to investigate whether, in the semilinear case, we may have a Nash equilibrium. Let us show that the answer is positive at least when $F \in W^{2, \infty}(\mathbb{R})$.

Let $f \in L^{2}(\mathcal{O} \times(0, T))$ be given and let $\left(v^{1}, v^{2}\right)$ be the associated Nash quasi-equilibrium. Note that, for any $s \in \mathbb{R}$ and $\left(w^{1}, w^{2}\right) \in \mathcal{H}$,

$$
\begin{aligned}
& \left\langle D_{1} J_{1}\left(f ; v^{1}+s w^{1}, v^{2}\right), w^{2}\right\rangle-\left\langle D_{1} J_{1}\left(f ; v^{1}, v^{2}\right), w^{2}\right\rangle=s \mu_{1} \iint_{\mathcal{O}_{1} \times(0, T)} w^{1} w^{2} \mathrm{~d} x \mathrm{~d} t \\
& +\alpha_{1} \iint_{\mathcal{O}_{d} \times(0, T)}\left(y^{s}-y_{1, d}\right) p^{s} \mathrm{~d} x \mathrm{~d} t-\alpha_{1} \iint_{\mathcal{O}_{d} \times(0, T)}\left(y-y_{1, d}\right) p \mathrm{~d} x \mathrm{~d} t,
\end{aligned}
$$

where

$$
\begin{cases}y_{t}^{s}-\Delta y^{s}+a(x, t) y^{s}=F\left(y^{s}\right)+f 1_{\mathcal{O}}+\left(v^{1}+s w^{1}\right) 1_{\mathcal{O}_{1}}+v^{2} 1_{\mathcal{O}_{2}} & \text { in } Q, \\ y^{s}=0 & \text { on } \Sigma, \\ y^{s}(x, 0)=y_{0} & \text { in } \Omega,\end{cases}
$$

$p^{s}$ is the derivative of $y^{s}$ with respect to $v^{1}$ in the direction $w^{2}$, i.e. the solution to

$$
\begin{cases}p_{t}^{s}-\Delta p^{s}+a(x, t) p^{s}=F^{\prime}\left(y^{s}\right) p^{s}+w^{2} 1_{\mathcal{O}_{1}} & \text { in } Q \\ p^{s}=0 & \text { on } \Sigma \\ p^{s}(x, 0)=0 & \text { in } \Omega\end{cases}
$$

and we have used the notation $y=\left.y^{s}\right|_{s=0}$ and $p=\left.p^{s}\right|_{s=0}$.

Let us introduce the adjoint of (3.11)

$$
\begin{cases}-\phi_{t}^{s}-\Delta \phi^{s}+a(x, t) \phi^{s}=F^{\prime}\left(y^{s}\right) \phi^{s}+\alpha_{1}\left(y^{s}-y_{1, d}\right) 1_{\mathcal{O}_{d}} & \text { in } Q \\ \phi^{s}=0 & \text { on } \Sigma, \\ \phi^{s}(x, T)=0 & \text { in } \Omega\end{cases}
$$

and let us also set $\phi=\left.\phi^{s}\right|_{s=0}$.

Replacing (3.12) into (3.9) and using integration by parts, we obtain the following identity:

$$
\begin{gathered}
\left\langle D_{1} J_{1}\left(f ; v^{1}+s w^{1}, v^{2}\right), w^{2}\right\rangle-\left\langle D_{1} J_{1}\left(f ; v^{1}, v^{2}\right), w^{2}\right\rangle=s \mu_{1} \iint_{\mathcal{O}_{1} \times(0, T)} w^{1} w^{2} \mathrm{~d} x \mathrm{~d} t \\
+\iint_{\mathcal{O}_{d} \times(0, T)}\left(\phi^{s}-\phi\right) w^{2} \mathrm{~d} x \mathrm{~d} t .
\end{gathered}
$$

Notice that

$$
\begin{aligned}
& -\left(\phi^{s}-\phi\right)_{t}-\Delta\left(\phi^{s}-\phi\right)+a(x, t)\left(\phi^{s}-\phi\right) \\
& \quad=\left[F^{\prime}\left(y^{s}\right)-F^{\prime}(y)\right] \phi^{s}+F^{\prime}(y)\left(\phi^{s}-\phi\right)+\alpha_{1}\left(y^{s}-y\right) 1_{\mathcal{O}_{d}} .
\end{aligned}
$$

Consequently, the limits

$$
\eta=\lim _{s \rightarrow 0} \frac{1}{s}\left(\phi^{s}-\phi\right) \text { and } h=\lim _{s \rightarrow 0} \frac{1}{s}\left(y^{s}-y\right)
$$

exist and satisfy

$$
\begin{cases}-\eta_{t}-\Delta \eta+a(x, t) \eta=F^{\prime \prime}(y) h \phi+F^{\prime}(y) \eta+\alpha_{1} h 1_{\mathcal{O}_{d}} & \text { in } Q, \\ h_{t}-\Delta h+a(x, t) h=F^{\prime}(y) h+w^{1} 1_{\mathcal{O}_{1}} & \text { in } Q, \\ \eta=h=0, & \text { on } \Sigma, \\ \eta(\cdot, T)=h(\cdot, 0)=0 & \text { in } \Omega\end{cases}
$$

Thus, from (3.13), we deduce that

$$
\left\langle D_{1}^{2} J_{1}\left(f ; v^{1}, v^{2}\right),\left(w^{1}, w^{2}\right)\right\rangle=\mu_{1} \iint_{\mathcal{O}_{1} \times(0, T)} w^{1} w^{2} \mathrm{~d} x \mathrm{~d} t+\iint_{\mathcal{O}_{1} \times(0, T)} \eta w^{2} \mathrm{~d} x \mathrm{~d} t .
$$


In particular, for all $w^{1} \in L^{2}\left(\mathcal{O}_{1} \times(0, T)\right)$, one has

$$
\left\langle D_{1}^{2} J_{1}\left(f ; v^{1}, v^{2}\right),\left(w^{1}, w^{1}\right)\right\rangle=\mu_{1} \iint_{\mathcal{O}_{1} \times(0, T)}\left|w^{1}\right|^{2} \mathrm{~d} x \mathrm{~d} t+\iint_{\mathcal{O}_{1} \times(0, T)} \eta w^{1} \mathrm{~d} x \mathrm{~d} t .
$$

Let $M>0$ be such that $\left|F^{\prime \prime}(s)\right| \leq M$ a.e. in $\mathbb{R}$. Let us show that, for some $C$ only depending on $\Omega, \mathcal{O}, T$, $\mathcal{O}_{i}, \mathcal{O}_{d}, \alpha_{i}, M, K,\|a\|_{L^{\infty}(\Omega)}$ and $\left\|y_{0}\right\|$, we have

$$
\left|\iint_{\mathcal{O}_{1} \times(0, T)} \eta w^{1} \mathrm{~d} x \mathrm{~d} t\right| \leq C\left(1+\|f\|_{L^{2}(\mathcal{O} \times(0, T))}\right)\left\|w^{1}\right\|_{\mathcal{H}_{1}}, \quad \forall w^{1} \in L^{2}\left(\mathcal{O}_{1} \times(0, T)\right) .
$$

In fact, from standard energy estimates, since $F^{\prime} \in L^{\infty}(Q)$, we have

$$
\int_{\Omega}|h(x, t)|^{2} \mathrm{~d} x+\iint_{Q}|\nabla h|^{2} \mathrm{~d} x \leq C \iint_{\mathcal{O}_{1} \times(0, T)}\left|w^{1}\right|^{2} \mathrm{~d} x \mathrm{~d} t .
$$

Using the PDEs in (3.13), we also get the following:

$$
\begin{aligned}
\iint_{\mathcal{O}_{1} \times(0, T)} \eta w^{1} \mathrm{~d} x \mathrm{~d} t & =\iint_{Q}\left(h_{t}-\Delta h+a(x, t) h-F^{\prime}(y) h\right) \eta \mathrm{d} x \mathrm{~d} t \\
& =\iint_{Q} h\left(-\eta_{t}-\Delta \eta+a(x, t) \eta-F^{\prime}(y) \eta\right) \mathrm{d} x \mathrm{~d} t \\
& =\iint_{Q}\left(F^{\prime \prime}(y) h \phi+\alpha_{1} h 1_{\mathcal{O}_{d}}\right) h \mathrm{~d} x \mathrm{~d} t \\
& =\iint_{Q}\left(F^{\prime \prime}(y)|h|^{2} \phi+\alpha_{1}|h|^{2} 1_{\mathcal{O}_{d}}\right) \mathrm{d} x \mathrm{~d} t .
\end{aligned}
$$

Let us first assume that $y_{0} \in H_{0}^{1}(\Omega)$. The idea is to find $r$ and $s$ such that

$$
\phi \in L^{r}\left(0, T ; L^{s}(\Omega)\right) \text { and } h \in L^{2 r^{\prime}}\left(0, T ; L^{2 s^{\prime}}(\Omega)\right),
$$

where $r^{\prime}$ and $s^{\prime}$ are the conjugate of $r$ and $s$, respectively. This will make possible to bound from above the last integral in (3.16).

It is clear that $h \in L^{2}\left(0, T ; H^{2}(\Omega)\right) \cap L^{\infty}\left(0, T ; H_{0}^{1}(\Omega)\right)$. For this reason, it is natural to ask for which values of $\alpha$ and $\beta$ the following embedding holds:

$$
L^{2}\left(0, T ; H^{2}(\Omega)\right) \cap L^{\infty}\left(0, T ; H_{0}^{1}(\Omega)\right) \hookrightarrow L^{\alpha}\left(0, T ; L^{\beta}(\Omega)\right) .
$$

By interpolation, we have that, for each $0<\theta<1$, (3.18) holds when

$$
\frac{1}{\alpha}=\frac{\theta}{2} \text { and } \frac{1}{\beta}=\frac{(N-4) \theta}{2 N}+\frac{(N-2)(1-\theta)}{2 N}=\frac{\alpha(N-2)-4}{2 \alpha N} .
$$

Taking $\alpha=2 r^{\prime}$ and $\beta=2 s^{\prime}$, we conclude that $r=\alpha /(\alpha-2)$ and $s=\alpha N / 2(\alpha+2)$.

Analogously, we have that $y \in L^{2}\left(0, T ; H^{2}(\Omega)\right) \cap L^{\infty}\left(0, T ; H_{0}^{1}(\Omega)\right) \hookrightarrow L^{a}\left(0, T ; L^{b}(\Omega)\right)$, with $b=2 a N /(a(N-$ $2)-4)$. Using the regularity results of the heat equation and the fact that $y_{i, d} \in L^{\infty}\left(\mathcal{O}_{i, d} \times(0, T)\right)$, it follows that

$$
\phi \in L^{a}\left(0, T ; W^{2, b}(\Omega)\right) \hookrightarrow L^{a}\left(0, T ; L^{\frac{N b}{N-2 b}}(\Omega)\right)=L^{a}\left(0, T ; L^{\frac{2 a N}{a N-6 a-4}}(\Omega)\right) .
$$

If $a=r=\alpha /(\alpha-2)$, we get $\phi \in L^{r}\left(0, T ; L^{\frac{2 \alpha N}{\alpha N-10 \alpha+8}}(\Omega)\right)$. To finish, we must have $L^{\frac{2 \alpha N}{\alpha N-10 \alpha+8}}(\Omega) \hookrightarrow L^{s}(\Omega)$, which is equivalent to

$$
\frac{\alpha N}{2(\alpha+2)} \leq \frac{2 \alpha N}{\alpha(N-10)+8}
$$

Thus, we see that this inequality holds true if and only if $N \leq 14$. 
From (3.2), (3.3), (3.10) for $s=0,(3.12)$ for $s=0,(3.16)$ and the estimates at Section 3.2, we see that, if $y_{0} \in H_{0}^{1}(\Omega)$ and $N \leq 14$,

$$
\begin{aligned}
\left|\iint_{\mathcal{O}_{1} \times(0, T)} \eta w^{1} \mathrm{~d} x \mathrm{~d} t\right| \leq & M\|h\|_{L^{2 r^{\prime}}\left(0, T ; L^{2 s^{\prime}}(\Omega)\right)}^{2}\|\phi\|_{L^{r}\left(0, T ; L^{s}(\Omega)\right)} \\
& +\alpha_{1}\|h\|_{L^{2}\left(\mathcal{O}_{d} \times(0, T)\right)}^{2} \leq \\
\leq & C\left(\|\phi\|_{L^{r}\left(0, T ; L^{s}(\Omega)\right)}+1\right)\left\|w^{1}\right\|_{\mathcal{H}_{1}}^{2} \\
\leq & C\left(\|y\|_{L^{2}(Q)}+1\right)\left\|w^{1}\right\|_{\mathcal{H}_{1}}^{2} \\
\leq & C\left(\sum_{i=1}^{2} \frac{1}{\mu_{i}}\left\|\phi^{i}\right\|_{\mathcal{H}_{i}}+\|f\|+\left\|y_{0}\right\|+1\right)\left\|w^{1}\right\|_{\mathcal{H}_{1}}^{2} \\
\leq & C(1+\|f\|)\left\|w^{1}\right\|_{\mathcal{H}_{1}}^{2} .
\end{aligned}
$$

This proves (3.15) in this case.

Now, let us assume that we only have $y_{0} \in L^{2}(\Omega)$. As in the first situation, the idea is to find $r$ and $s$ such that (3.17) holds. Since the regularity of $\eta$ does not depend on the data $y_{0}$, we still have $\eta \in L^{2}\left(0, T ; H^{2}(\Omega)\right) \cap$ $L^{\infty}\left(0, T ; H_{0}^{1}(\Omega)\right)$ and, therefore, $\eta \in L^{\alpha}\left(0, T ; L^{\beta}(\Omega)\right)$, where $\alpha$ and $\beta$ are as above. In this case, we have by a interpolation argument that $y \in L^{2}\left(0, T ; H_{0}^{1}(\Omega)\right) \cap L^{\infty}\left(0, T ; L^{2}(\Omega)\right) \hookrightarrow L^{\bar{a}}\left(0, T ; L^{\bar{b}}(\Omega)\right)$, where $\bar{a} \geq 2$ and $\bar{b}=2 N \bar{a} /(\bar{a} N-4)$. Using again parabolic regularity, we get

$$
\phi \in L^{\bar{a}}\left(0, T ; W^{2, \bar{b}}(\Omega)\right) \hookrightarrow L^{\bar{a}}\left(0, T ; L^{\frac{N \bar{b}}{N-2 b}}(\Omega)\right)=L^{\bar{a}}\left(L^{\frac{2 \bar{a} N}{a(N-4)-4}}(\Omega)\right) .
$$

If $\bar{a}=r=\alpha /(\alpha-2)$, we have $\phi \in L^{r}\left(L^{\frac{2 \alpha N}{\alpha(N-8)+8}}(\Omega)\right)$. Now, to finish the proof, we must have $L^{\frac{2 \alpha N}{\alpha(N-8)+8}}(\Omega) \hookrightarrow$ $L^{s}(\Omega)$, which is equivalent to

$$
\frac{\alpha N}{2(\alpha+2)} \leq \frac{2 \alpha N}{\alpha(N-8)+8}
$$

Since this holds if and only if $N \leq 12$, the estimate (3.15) is also proved in this case.

Taking into account (3.14) and (3.15), we see that

$$
\left\langle D_{1}^{2} J_{1}\left(f ; v^{1}, v^{2}\right),\left(w^{1}, w^{1}\right)\right\rangle \geq\left(\mu_{1}-C\left(1+\|f\|_{L^{2}(\mathcal{O} \times(0, T))}\right)\right)\left\|w^{1}\right\|_{\mathcal{H}_{1}}^{2} \mathrm{~d} x \mathrm{~d} t .
$$

Note that the previous constant $C$ can be chosen independent of $\mu_{1}$ and $\mu_{2}$.

In a similar way, it can be shown that, under the previous assumptions on $y_{0}$ and $N$,

$$
\left\langle D_{2}^{2} J_{2}\left(f ; v^{1}, v^{2}\right),\left(w^{2}, w^{2}\right)\right\rangle \geq\left(\mu_{2}-C\left(1+\|f\|_{L^{2}(\mathcal{O} \times(0, T))}\right)\right)\left\|w^{2}\right\|_{\mathcal{H}_{2}}^{2} \mathrm{~d} x \mathrm{~d} t .
$$

for another constant $C$ independent of $\mu_{1}$ and $\mu_{2}$.

It is now clear that, for sufficiently large $\mu_{1}$ and $\mu_{2}$, the couple $\left(v^{1}, v^{2}\right)$ is a Nash equilibrium in the sense of (1.4).

\section{The CASE With Restrictions}

In this section, we will prove Theorem 1.5.

We return to the Stackelberg-Nash null controllability problem for a linear parabolic PDE, but we impose some restrictions: the followers $\left(v^{1}, v^{2}\right)$ are supposed to minimize the functionals (1.2) subject to the convex constraints $v^{i} \in \mathcal{U}_{i}(i=1,2)$, where the $\mathcal{U}_{i}$ are given by (1.12). This is a more difficult problem. The search of a pair $\left(v^{1}, v^{2}\right)$ satisfying (1.4), where the minimizations are performed in $\mathcal{U}_{1, d}$ and $\mathcal{U}_{2, d}$, is equivalent to the following:

$$
D_{1} J_{1}\left(f ; v^{1}, v^{2}\right)\left(\hat{v}^{1}-v^{1}, 0\right) \geq 0, \quad \forall \hat{v}^{1} \in \mathcal{U}_{1, d}, \quad v^{1} \in \mathcal{U}_{1, d}
$$


and

$$
D_{2} J_{2}\left(f ; v^{1}, v^{2}\right)\left(0, \hat{v}^{2}-v^{2}\right) \geq 0, \quad \forall \hat{v}^{2} \in \mathcal{U}_{2, d}, \quad v^{2} \in \mathcal{U}_{2, d} .
$$

As in Section 2, with the change of variable $z=y-\bar{y}$, we are led to a null controllability problem. Then, we see that (4.1)-(4.2) is equivalent to

$$
\left\{\begin{array}{l}
\alpha_{i} \iint_{\mathcal{O}_{i, d} \times(0, T)}\left(z-z_{i, d}\right) w^{i} \mathrm{~d} x \mathrm{~d} t+\mu_{i} \iint_{\mathcal{O}_{i} \times(0, T)} v^{i}\left(\hat{v}^{i}-v^{i}\right) \mathrm{d} x \mathrm{~d} t \geq 0, \\
\forall \hat{v}^{i} \in \mathcal{U}_{i, d}, \quad v^{i} \in \mathcal{U}_{i, d},
\end{array}\right.
$$

where $w^{i}$ is the derivative of $z$ with respect to $\hat{v}^{i}$ in the direction $v^{i}$, that is to say, the solution to

$$
\begin{cases}w_{t}^{i}-\Delta w^{i}+a(x, t) w^{i}=v^{i} 1_{\mathcal{O}_{i}} & \text { in } Q, \\ w^{i}=0 & \text { on } \Sigma, \\ w^{i}(\cdot, 0)=0 & \text { in } \Omega .\end{cases}
$$

The adjoint system associated to (4.4) is given by

$$
\begin{cases}-\phi_{t}^{i}-\Delta \phi^{i}+a(x, t) \phi^{i}=\alpha_{i}\left(z-z_{i, d}\right) 1_{\mathcal{O}_{i, d}} & \text { in } Q, \\ \phi=0 & \text { on } \Sigma, \\ \phi(\cdot, T)=0 & \text { in } \Omega .\end{cases}
$$

Replacing the equation satisfied by $\phi^{i}$ in (4.3), we obtain

$$
\iint_{\mathcal{O}_{i} \times(0, T)}\left(\phi^{i}+\mu_{i} v^{i}\right)\left(\hat{v}^{i}-v^{i}\right) \mathrm{d} x \mathrm{~d} t \geq 0, \quad \forall \hat{v}^{i} \in \mathcal{U}_{i, d}, \quad v^{i} \in \mathcal{U}_{i, d}, \quad i=1,2 .
$$

Now, by introducing the projectors $\mathbb{P}_{\mathcal{U}_{i, d}}: L^{2}\left(\mathcal{O}_{i} \times(0, T)\right) \rightarrow \mathcal{U}_{i, d}$, we see that $(4.5)$ can be rewritten equivalently in the form

$$
v^{i}=\mathbb{P}_{\mathcal{U}_{i, d}}\left(-\left.\frac{1}{\mu_{i}} \phi^{i}\right|_{\mathcal{O}_{i} \times(0, T)}\right), \quad i=1,2 .
$$

We may group all this information to get the following system:

$$
\begin{cases}z_{t}-\Delta z+a(x, t) z=f 1_{\mathcal{O}}+\sum_{i=1}^{2} \mathbb{P}_{\mathcal{U}_{i, d}}\left(-\left.\frac{1}{\mu_{i}} \phi^{i}\right|_{\mathcal{O}_{i} \times(0, T)}\right) & \text { in } Q, \\ -\phi_{t}^{i}-\Delta \phi^{i}+a(x, t) \phi^{i}=\alpha_{i}\left(z-z_{i, d}\right) 1_{\mathcal{O}_{i, d}} & \text { in } Q, \\ z=0, \quad \phi^{i}=0 & \text { on } \Sigma, \\ z(\cdot, 0)=z^{0}, \quad \phi^{i}(\cdot, T)=0 & \text { in } \Omega .\end{cases}
$$

Let us prove that, under the assumptions (2.7), for each $f \in L^{2}(\mathcal{O} \times(0, T))$ there exists exactly one solution to (4.6), i.e. there exists a unique Nash equilibrium $\left(v^{1}, v^{2}\right)$ in $\mathcal{U}_{1, d} \times \mathcal{U}_{2, d}$. Indeed, notice that (4.5) can also be rewritten in the form

$$
\left\{\begin{array}{l}
\left(\mathbb{L}\left(v^{1}, v^{2}\right),\left(\hat{v}^{1}, \hat{v}^{2}\right)-\left(v^{1}, v^{2}\right)\right) \geq\left(\Psi,\left(\hat{v}^{1}, \hat{v}^{2}\right)-\left(v^{1}, v^{2}\right)\right)_{\mathcal{H}} \\
\forall\left(v^{1}, v^{2}\right) \in \mathcal{U}_{1, d} \times \mathcal{U}_{2, d}, \quad\left(\hat{v}^{1}, \hat{v}^{2}\right) \in \mathcal{U}_{1, d} \times \mathcal{U}_{2, d}
\end{array}\right.
$$

where $\mathbb{L}$ and $\Psi$ are respectively given by (2.4) and (2.6). If $\mu_{1}$ and $\mu_{2}$ satisfy (2.7), $\mathbb{L}$ is a coercive continuous bilinear form on $\mathcal{H}$, whence (4.7) is uniquely solvable. Furthermore, it is clear that the couple $\left(v^{1}, v^{2}\right)$ and the associated state $z$ satisfy (again) the estimates (2.9) and (2.10). As in the semilinear case, we will analyze and solve the null controllability problem for (4.6) by a fixed-point method. To this end, note that the projectors $\mathbb{P}_{\mathcal{U}_{i, d}}$ are given as follows:

$$
\mathbb{P}_{\mathcal{U}_{i, d}}(k)(x, t)= \begin{cases}k(x, t) & \text { if } k(x, t) \in I_{i}, \\ P_{i}(k(x, t)) & \text { otherwise }\end{cases}
$$


for $(x, t)$ a.e. in $\mathcal{O}_{i} \times(0, T)$, where $P_{i}: \mathbb{R} \rightarrow I_{i}$ is the usual projector on the interval $I_{i}$. Also, note that, for every $k \in \mathcal{H}_{i}, \mathbb{P}_{\mathcal{U}_{i, d}}$ can be written in the form $\mathbb{P}_{\mathcal{U}_{i, d}}(k)=q_{i}(k) k$, where the function $k \mapsto q_{i}(k)$ is continuous on $\mathcal{H}_{i}$ and

$$
\left\|q_{i}(k)\right\|_{\infty} \leq C, \quad \forall k \in \mathcal{H}_{i} .
$$

Therefore, the controllability problem is reduced to find $f \in L^{2}(\mathcal{O} \times(0, T))$ such that the solution to

$$
\begin{cases}z_{t}-\Delta z+a(x, t) z=f 1_{\mathcal{O}}-\sum_{i=1}^{2} \tilde{q}_{i}\left(\phi^{i}\right) \phi^{i} 1_{\mathcal{O}_{i}} & \text { in } Q, \\ -\phi_{t}^{i}-\Delta \phi^{i}+a(x, t) \psi=\alpha_{i}\left(z-z_{i, d}\right) 1_{\mathcal{O}_{i, d}} & \text { in } Q, \\ z=0, \quad \phi^{i}=0 & \text { on } \Sigma, \\ z(\cdot, 0)=z^{0}, \quad \phi^{i}(\cdot, T)=0 & \text { in } \Omega,\end{cases}
$$

where $\tilde{q}_{i}\left(\phi^{i}\right)$ stands for the function $\tilde{q}_{i}\left(\phi^{i}\right)=q_{i}\left(-\left.\frac{1}{\mu_{i}} \phi^{i}\right|_{\mathcal{O}_{i} \times(0, T)}\right)$, satisfies $(2.2)$. But this can be done easily. Indeed, for each couple $\left(\tilde{\phi}^{1}, \tilde{\phi}^{2}\right) \in\left[L^{2}(Q)\right]^{2}$ we can consider the system

$$
\begin{cases}z_{t}-\Delta z+a(x, t) z=f 1_{\mathcal{O}}-\sum_{i=1}^{2} \tilde{q}_{i}\left(\tilde{\phi}^{i}\right) \phi^{i} 1_{\mathcal{O}_{i}} & \text { in } Q, \\ -\phi_{t}^{i}-\Delta \phi^{i}+a(x, t) \psi=\alpha_{i}\left(z-z_{i, d}\right) 1_{\mathcal{O}_{i, d}} & \text { in } Q, \\ z=0 \quad \phi^{i}=0 & \text { on } \Sigma, \\ z(\cdot, 0)=z^{0}, \quad \phi^{i}(\cdot, T)=0 & \text { in } \Omega .\end{cases}
$$

The arguments in Sections 2.2 and 3.2 can be applied again to (4.9). The main consequence is that there exists exactly one minimal $L^{2}$ norm null control $f$ for this system with

$$
\|f\|_{L^{2}(\mathcal{O} \times(0, T))} \leq C
$$

and, also, $z, \phi^{1}$ and $\phi^{2}$ uniformly bounded in $L^{2}\left(0, T ; H_{0}^{1}(\Omega)\right) \cap L^{\infty}\left(0, T ; L^{2}(\Omega)\right)$ and $z_{t}, \phi_{t}^{1}$ and $\phi_{t}^{2}$ uniformly bounded (at least) in $L^{2}\left(0, T ; H^{-1}(\Omega)\right)$. Hence, it is not difficult to deduce that the mapping $\left(\tilde{\phi}^{1}, \tilde{\phi}^{2}\right) \mapsto\left(\phi^{1}, \phi^{2}\right)$ possesses at least one fixed-point. Such a fixed-point satisfies, together with some $f$ and some $z,(4.8)$ and (2.2). This concludes the proof of Theorem 1.5.

\section{Some AdDitional COMments And QUeStions}

\subsection{On the assumption $\mathcal{O}_{1, \mathrm{~d}}=\mathcal{O}_{2, \mathrm{~d}}$}

The assumption (1.10) is used in (2.20) and only there. Indeed, in combination with (2.21) and (2.22), (2.20) yields (2.23). At present, we do not know whether an estimate like (2.13) remains true for $\mathcal{O}_{1, d} \neq \mathcal{O}_{2, d}$. However, this is the case if we modify appropriately the secondary functionals $J_{i}$. In fact, let $\rho_{*}=\rho_{*}(x, t)$ be a weight (a positive continuous function on $\bar{\Omega} \times(0, T))$ such that $\rho_{*} \geq \mathrm{e}^{s \sigma / 2}$, see (2.18). We assume now that the followers produce a Nash equilibrium with respect to the functionals

$$
\tilde{J}_{i}\left(f ; v^{1}, v^{2}\right):=\frac{\alpha_{i}}{2} \iint_{\mathcal{O}_{i, d} \times(0, T)}\left|y-y_{i, d}\right|^{2} \mathrm{~d} x \mathrm{~d} t+\frac{\mu_{i}}{2} \iint_{\mathcal{O}_{i} \times(0, T)} \rho_{*}^{2}\left|v^{i}\right|^{2} \mathrm{~d} x \mathrm{~d} t, \quad i=1,2 .
$$

With computations similar to those in Section 2.1, we obtain the following optimality system:

$$
\begin{cases}z_{t}-\Delta z+a(x, t) z=f 1_{\mathcal{O}}-\sum_{i=1}^{2} \frac{1}{\mu_{i}} \rho_{*}^{-2} \phi^{i} 1_{\mathcal{O}_{i}} & \text { in } Q, \\ -\phi_{t}^{i}-\Delta \phi^{i}+a(x, t) \phi^{i}=\alpha_{i}\left(z-z_{i, d}\right) 1_{\mathcal{O}_{i, d}} & \text { in } Q, \\ y=0, \quad \phi^{i}=0 & \text { in } \Sigma, \\ y(\cdot, 0)=y^{0}, \quad \phi^{i}(\cdot, T)=0 & \text { in } \Omega .\end{cases}
$$


The associated adjoint system is given by

$$
\begin{cases}-\psi_{t}-\Delta \psi+a(x, t) \psi=\sum_{i=1}^{2} \alpha_{i} \gamma^{i} 1_{\mathcal{O}_{i, d}} & \text { in } Q, \\ \gamma_{t}^{i}-\Delta \gamma^{i}+a(x, t) \gamma^{i}=-\frac{1}{\mu_{i}} \rho_{*}^{-2} \psi 1_{\mathcal{O}_{i}} & \text { in } Q, \\ \psi=0, \quad \gamma^{i}=0 & \text { on } \Sigma, \\ \psi(\cdot, T)=\psi^{T}, \quad \gamma^{i}(\cdot, 0)=0 & \text { in } \Omega\end{cases}
$$

and the main task is to prove an estimate like (2.13) for the solutions $\left(\psi, \gamma^{1}, \gamma^{2}\right)$. In this situation, we have an useful energy inequality for the $\gamma^{i}$ :

$$
\left\|\gamma^{i}(\cdot, \tau)\right\|^{2}+\int_{0}^{\tau}\left\|\nabla \gamma^{i}(\cdot, t)\right\|^{2} \mathrm{~d} t \leq \frac{C}{\mu_{i}^{2}} \iint_{Q} \rho_{*}^{-4}|\psi|^{2} \mathrm{~d} x \mathrm{~d} t .
$$

Using (5.1) in the right-hand side of $(2.20)$, since the $\mu_{i}$ are sufficiently large, we get

$$
I_{3}(\psi) \leq C s^{3} \lambda^{4} \iint_{\omega \times(0, T)} \mathrm{e}^{-2 s \alpha} \xi^{3}|\psi|^{2} \mathrm{~d} x \mathrm{~d} t .
$$

Combining (5.2) and (2.26), we arrive at (2.13). This shows that if we replace $J_{i}$ by $\tilde{J}_{i}(i=1,2)$, the claims in Theorem 1.1 to 1.5 remain true. In fact, this is not surprising: if we impose $\tilde{J}_{i}<+\infty$, then we force the controls $v^{i}$ to vanish exponentially as $t \rightarrow T^{-}$and the leader $f$ finds no obstruction to control the system.

As mentioned above, it is unknown whether (2.13) continues to be true in the original framework (1.2) when $\mathcal{O}_{1, d} \neq \mathcal{O}_{2, d}$.

\subsection{Stackelberg-Nash controllability and Stokes and Navier-Stokes systems}

It makes complete sense to consider the Stokes-like system

$$
\begin{cases}y_{t}-\Delta y+(w \cdot \nabla) y+\nabla p=f 1_{\mathcal{O}}+v^{1} 1_{\mathcal{O}_{1}}+v^{2} 1_{\mathcal{O}_{2}} & \text { in } Q, \\ \nabla \cdot y=0 & \text { in } Q, \\ y=0 & \text { on } \Sigma, \\ y(\cdot, 0)=y^{0} & \text { in } \Omega,\end{cases}
$$

where $\Omega, T, \mathcal{O}$ and the $\mathcal{O}_{i}$ are as above, $y^{0}$ belongs to the Hilbert space

$$
H:=\left\{z \in L^{2}(\Omega)^{N}: \nabla \cdot z=0 \text { in } \Omega, z \cdot n=0 \text { on } \Gamma\right\},
$$

the field $w$ belongs to $L^{\infty}(0, T ; H)$ and the controls $f$ and $v^{i}$ satisfy

$$
f \in L^{2}(\mathcal{O} \times(0, T))^{N}, \quad v^{i} \in L^{2}\left(\mathcal{O}_{i} \times(0, T)\right)^{N} .
$$

With functionals $J$ and $J_{i}$ similar to those in the previous sections, we can formulate again the StackelbergNash null controllability problem for (5.3). Results of the same kind can be obtained easily by adapting the arguments in Sections 2 to 4.

The situation is obviously more difficult to analyze when we consider the Navier-Stokes system

$$
\begin{cases}y_{t}-\Delta y+(y \cdot \nabla) y+\nabla p=f 1_{\mathcal{O}}+v^{1} 1_{\mathcal{O}_{1}}+v^{2} 1_{\mathcal{O}_{2}} & \text { in } Q, \\ \nabla \cdot y=0 & \text { in } Q, \\ y=0 & \text { on } \Sigma, \\ y(\cdot, 0)=y^{0} & \text { in } \Omega .\end{cases}
$$

Now, the existence of Nash equilibria or quasi-equilibria for each $f$ and, of course, whether or not there exist null controls and associated Nash equilibrium pairs are open problems.

For other controllability results for Stokes and Navier-Stokes systems, see [6, 8-10,12]. 


\subsection{Other Stackelberg strategies}

It is possible to introduce other strategies to control systems of the kind (1.1). One of them is the so called Stackelberg-Pareto method.

For each $f \in L^{2}(\mathcal{O} \times(0, T))$, we can associate one or several Pareto equilibrium pairs $\left(u^{1}(f), u^{2}(f)\right) \in \mathcal{H}$. By definition, this means that there is no $\left(\hat{u}^{1}, \hat{u}^{2}\right) \in \mathcal{H}$ satisfying

$$
J_{i}\left(\hat{u}^{1}, \hat{u}^{2}\right) \leq J_{i}\left(u^{1}(f), u^{2}(f)\right), \quad i=1,2,
$$

one of these inequalities at least being strict. Then, we search for $f$ such that the states $y$ associated to $f$ and the $\left(u^{1}(f), u^{2}(f)\right)$ satisfy $(1.9)$, where $\bar{y}=\bar{y}(x, t)$ is a prescribed uncontrolled solution to (1.1).

The analysis of Stackelberg-Pareto controllability will be the goal of a forthcoming paper.

\subsection{The boundary case}

It is natural to try to prove results similar to Theorems 1.1, 1.3 and 1.5 with boundary controls. For instance, let us consider the system

$$
\begin{cases}z_{t}-\Delta z+a(x, t) z=0 & \text { in } Q, \\ z=f 1_{\mathcal{S}}+v^{1} 1_{\mathcal{S}_{1}}+v^{2} 1_{\mathcal{S}_{2}} & \text { on } \Sigma, \\ z(\cdot, 0)=z^{0} & \text { in } \Omega,\end{cases}
$$

where $\mathcal{S}, \mathcal{S}_{1}, \mathcal{S}_{2} \subset \Gamma$ are non-empty closed sets and let us introduce the functionals

$$
L_{i}\left(f ; v^{1}, v^{2}\right):=\frac{\alpha_{i}}{2} \iint_{\mathcal{O}_{i, d} \times(0, T)}\left|z-z_{i, d}\right|^{2} \mathrm{~d} x \mathrm{~d} t+\frac{\mu_{i}}{2} \iint_{\mathcal{S}_{i} \times(0, T)}\left|v^{i}\right|^{2} \mathrm{~d} \Gamma \mathrm{d} t, \quad i=1,2 .
$$

Now, the problem is to find for each $f$ a Nash equilibrium $\left(v^{1}(f), v^{2}(f)\right)$ associated to the functionals $L_{i}$ and, then, choose $f$ in a appropriate way such that $z(x, T) \equiv 0$.

We can try to solve this problem as before. However, we find some technical difficulties, as shown below.

Arguing as in Section 2, we see that the optimality system for $\left(v^{1}(f), v^{2}(f)\right)$ is the following:

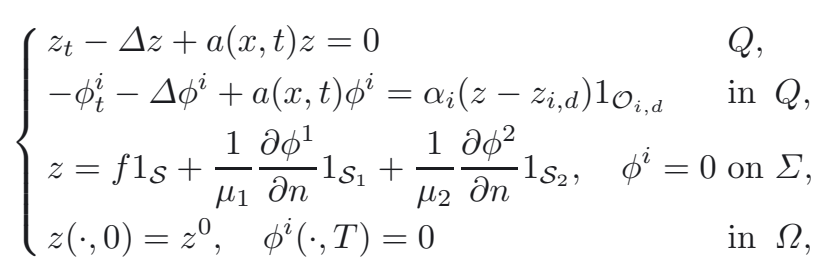

where $n=n(x)$ is the outward unit normal to $\Omega$ at the point $x \in \Gamma$. The corresponding adjoint is given by

$$
\begin{cases}-\psi_{t}-\Delta \psi+a(x, t) \psi=\sum_{i=1}^{2} \alpha_{i} \gamma^{i} 1_{\mathcal{O}_{i, d}} & \text { in } Q, \\ \gamma_{t}^{i}-\Delta \gamma^{i}+a(x, t) \gamma^{i}=0 & \text { in } Q, \\ \psi=0, \quad \gamma^{i}=\frac{1}{\mu_{i}} \psi 1_{\mathcal{S}_{i}} & \text { on } \Sigma, \\ \psi(\cdot, T)=\psi^{T}, \quad \gamma^{i}(\cdot, 0)=0 & \text { in } \Omega .\end{cases}
$$

Thus, if we try to adapt the proof of Proposition 2.2, we see at once that the following conditions are required:

$$
\mathcal{O}_{1, d}=\mathcal{O}_{2, d}=\mathcal{O}_{d} \quad \text { and } \quad \overline{\mathcal{O}_{d}} \cap \mathcal{S} \neq \emptyset .
$$

The main difficulty in this case is that we have to combine a boundary Carleman inequality for $\psi$ and a distributed Carleman inequality for $h=\alpha_{1} \gamma^{1}+\alpha_{2} \gamma^{2}$ for functions satisfying nonhomogeneous Dirichlet boundary conditions on $\Sigma$. This interesting situation will be also analyzed in a forthcoming paper. 
Acknowledgements. This paper was written in part during several visits of the first and the third authors to the Institute of Mathematics of the University of Sevilla (IMUS). The authors are indebted to this Institute for its assistance.

\section{REFERENCES}

[1] H. Brézis, Analyse Fonctionnelle, Théorie et Applications. Dunod, Paris (1999).

[2] J.C. Cox and M. Rubinstein, Options Markets. Prentice-Hall. Englewood Cliffs, NJ (1985).

[3] J.I. Díaz, On the von Neumann problem and the approximate controllability of Stackelberg-Nash strategies for some environmental problems. Rev. R. Acad. Cien., Ser. A. Math. 96 (2002) 343-356.

[4] J.I. Díaz and J.-L. Lions, On the approximate controllability of Stackelberg-Nash strategies. Ocean circulation and pollution control: a mathematical and numerical investigation, Madrid, 1997. Springer, Berlin (2004) 17-27.

[5] E. Fernández-Cara and S. Guerrero, Global Carleman inequalities for parabolic systems and applications to controllability. SIAM J. Control Optim. 45 (2006) 1395-1446.

[6] E. Fernández-Cara, S. Guerrero, O.Y. Imanuvilov and J.-P. Puel, Local exact controllability of the Navier-Stokes system. J. Math. Pures Appl. 83 (2004) 1501-1542.

[7] A.V. Fursikov and O.Y. Imanuvilov, Controllability of evolution equations. Vol. 34 of Lecture Note Series. Research Institute of Mathematics, Seoul National University, Seoul (1996).

[8] A.V. Fursikov and O.Y. Imanuvilov, Exact controllability of the Navier-Stokes and Boussinesq equations. Russian Math. Surveys 54 (1999) 565-618.

[9] M. González-Burgos, S. Guerrero and J.-P. Puel, Local exact controllability to the trajectories of the Boussinesq system via a fictitious control on the divergence equation. Commun. Pure Appl. Anal. 8 (2009) 311-333.

[10] S. Guerrero, O.Y. Imanuvilov and J.-P. Puel, A result concerning the global approximate controllability of the Navier-Stokes system in dimension 3. J. Math. Pures Appl. 98 (2012) 689-709.

[11] F. Guillén-González, F.P. Marques-Lopes and M.A. Rojas-Medar, On the approximate controllability of Stackelberg-Nash strategies for Stokes equations. Proc. Amer. Math. Soc. 141 (2013) 1759-1773.

[12] O.Y. Imanuvilov, Remarks on exact controllability for the Navier-Stokes equations. ESAIM Control Optim. Calc. Var. 6 (2001) 39-72.

[13] O.Y. Imanuvilov and M. Yamamoto, Carleman Estimate for a Parabolic Equation in a Sobolev Space of Negative Order and its Applications, Vol. 218 of Lect. Notes Pure Appl. Math. Dekker, New York (2001).

[14] J.-L. Lions, Contrôle de Pareto de systèmes distribués. Le cas d'évolution. C.R. Acad. Sci. Paris, Sér. I 302 (1986) $413-417$.

[15] J.-L. Lions, Some remarks on Stackelberg's optimization. Math. Models Methods Appl. Sci. 4 (1994) $477-487$.

[16] J.F. Nash, Noncooperative games. Ann. Math. 54 (1951) 286-295.

[17] V. Pareto, Cours d'économie politique. Rouge, Laussane, Switzerland (1896).

[18] A.M. Ramos, R. Glowinski and J. Periaux, Pointwise control of the Burgers equation and related Nash equilibria problems: A computational approach. J. Optim. Theory Appl. 112 (2001) 499-516.

[19] A.M. Ramos, R. Glowinski and J. Periaux, Nash equilibria for the multiobjective control of linear partial differential equations. J. Optim. Theory Appl. 112 (2002) 457-498.

[20] S.M. Ross, An introduction to mathematical finance. Options and other topics. Cambridge University Press, Cambridge (1999).

[21] H. Von Stalckelberg, Marktform und gleichgewicht. Springer, Berlin, Germany (1934).

[22] P. Wilmott, S. Howison and J. Dewynne, The mathematics of financial derivatives. Cambridge University Press, New York (1995).

[23] E. Zuazua, Exact controllability for the semilinear wave equation, J. Math. Pures Appl. 69 (1990) 1-31. 